MODELING, IDENTIFICATION AND CONTROL, 2001, VOL. 22, NO. 1, 29-52

doi:10.4173/mic.2001.1.3

\title{
Uncertainty Modeling and Robust Output Feedback Control of Nonlinear Discrete Systems: A Mathematical Programming Approach
}

\author{
OLAV SLUPPHAUG*†, LARS IMSLAND*\$ and \\ BJARNE A. FOSS*§
}

\begin{abstract}
Keywords: Robust control, Constrained control, Affine parameter-dependent models, Bilinear matrix inequalities, Semi-infinite programming. Nonlinear model predictive control.
\end{abstract}

\begin{abstract}
We present a mathematical programming approach to robust control of nonlinear systems with uncertain, possibly time-varying, parameters. The uncertain system is given by different local affine parameter dependent models in different parts of the state space. It is shown how this representation can be obtained from a nonlinear uncertain system by solving a set of continuous linear semi-infinite programming problems, and how each of these problems can be solved as a (finite) series of ordinary linear programs. Additionally, the system representation includes controland state constraints. The controller design method is derived from Lyapunov stability arguments and utilizes an affine parameter dependent quadratic Lyapunov function. The controller has a piecewise affine output feedback structure, and the design amounts to finding a feasible solution to a set of linear matrix inequalities combined with one spectral radius constraint on the product of two positive definite matrices. A local solution approach to this nonconvex feasibility problem is proposed. Complexity of the design method and some special cases such as statefeedback are discussed. Finally, an application of the results is given by proposing an on-line computationally feasible algorithm for constrained nonlinear statefeedback model predictive control with robust stability.
\end{abstract}

\section{Introduction}

Robust controller design is a key factor for implementing controllers. Robust design becomes particularly important, but also challenging, for nonlinear constrained uncertain systems with output feedback, the outset for this work.

Model uncertainty can be formulated in different ways; the most common being frequency-based model uncertainty (Skogestag and Postlethwaite 1996) and parameter-based uncertainty (Ackermann 1993). Frequency-based model uncertainty is used to describe model sets of transfer functions. There exist powerful techniques like $H_{\infty}$ design and structured singular values for robust control analysis and design based on this model description, see e.g. (Skogestag and Postlethwaite 1996). We focus on uncertain nonlinear state-space discrete-time systems formulated by piecewise affine

\footnotetext{
*Department of Engineering Cybernetics, Norwegian University of Science and Technology, N-7491 Trondheim, Norway.

†Post Doctoral Fellow. Presently at ABB Industri AS. E-mail: Olav.Slupphaug@no.abb.com

†Doctoral student. E-mail: Lars.Imsland@itk.ntnu.no

§rofessor. E-mail: Bjarne.A.Foss@itk.ntnu.no. Corresponding author.

Uncertainty modeling and robust output feedback of nonlinear discrete systems: A mathematical programming approach. Olav Slupphaug, Lars Imsland and Bjarne A. Foss. 2000 든 John Wiley \& Sons Limited. Reproduced, with permission, from International Journal of Robust and Nonlinear Control.
} 
parameter dependent models. The motivation for exploiting this model uncertainty class is the fact that it can be used to construct piecewise affine feedback controllers with robust stability guarantees using BMIs, cf. (Slupphaug and Foss 1999). Moreover, this enables the synthesis method to include input- and state constraints.

In this paper we emphasize the derivation of constructive methods, and utilize the powerful paradigm of mathematical programming, in particular methods for computing solutions to linear matrix inequality (LMI) and bilinear matrix inequality (BMI) feasibility problems as well as linear semi-infinite programming.

In LMI-based control, which has gained a lot of interest in the last few years, control system analysis- and synthesis problems are formulated as convex optimization problems involving LMIs (Boyd et al. 1994), (Scherer and Weiland 1999). The reason for this interest is the development of very efficient interior-point algorithms for solving such problems (Boyd et al. 1994), (Nesterov and Nemirovskii 1994), (Wolkowicz et al. 2000). Many interesting control problems, in particular robust control problems and multi-objective control problems, can be solved within the LMI framework. There are, however, interesting control problems that are very hard or impossible to formulate within the LMI framework. Some of these problems can be formulated as more general BMI problems (Goh et al. 1996). BMI problems are in general non-convex and hence much harder to solve than (convex) LMI problems. The efficient algorithms developed for LMI problems, however, provide a constructive basis for developing algorithms for solving BMI problems. Consult (Tuan et al. 2000) and (Goh et al. 1994) for global approaches. In this paper we obtain a specially structured BMI that we solve locally by means of an algorithm given in (El Ghaoui et al. 1997).

Model predictive control (MPC) is a methodology that has gained widespread industrial use (Qin and Badgwell 1997), especially linear MPC which can be viewed as a mature field. Applications of nonlinear MPC are also coming into use as reported in a newly published survey (Qin and Badgwell 1999). There exists a well developed theoretical foundation for analyzing linear MPC in the nominal case, see e.g. (Morari and Lee 1997) for a survey of this. There are also some results appearing for nonlinear MPC as well as for robust MPC as reviewed in (Allgöwer et al. 1999). The surveys (Morari and Lee 1997), (Allgöwer et al. 1999) and (Bemporad and Morari 1999) all suggest, however, that nonlinear- and robust MPC are areas open to significant future advances. In this paper we will show how the methodology developed herein can be used to guarantee stability for the robust and constrained nonlinear state-feedback MPC case with very low on-line computational demands.

The outline of the paper is as follows: In Section 2 we present the uncertainty class that is considered. It is comprised of a set of affine parameter dependent models, where each affine parameter dependent model is valid in some subset of the statespace. In Section 3 we show how the synthesis of a stabilizing piecewise affine static output feedback for this uncertainty class can be formulated as an LMI feasibility problem under a spectral radius constraint on the product of two positive definite matrices. The formulation is based on a quadratic parameter dependent Lyapunov function. The design problem can become quite complex, hence, complexity issues are discussed. Additionally, we show how to embed dynamic output feedback into the design method. Next, in Section 4, we present a local solution approach to the nonconvex feasibility problem. The derivation of the uncertainty class from a given nonlinear system with uncertain parameters is the topic of Section 5. We develop an algorithm and highlight its use by a simple example. In Section 6 we apply the control design method developed in Section 3 to obtain a nonlinear MPC controller with a 
robust stability guarantee. This result is limited to the state-feedback case. Finally we make some concluding remarks.

\section{Uncertainty characterization}

In the controller synthesis, cf. Section 3 , we assume that the uncertainty model is given as the following difference inclusion with an uncertain output mapping

$$
\left.\begin{array}{rl}
x_{k+1} & \in U_{i \in \mathscr{S}\left(x_{k}\right)} \mathscr{M}_{i}\left(x_{k}, u_{k}, \Theta\right) \\
y_{k} & \in \mathrm{U}_{i \in \mathscr{S}\left(x_{k}\right)} \mathscr{H}_{i}\left(x_{k}, \Theta\right)
\end{array}\right\} \forall k \in \mathbb{N}, x_{k} \in X_{m}, u_{k} \in U_{m}
$$

where $U_{m} \subset \mathbb{R}^{m}$ and $X_{m}:=U_{i \in I_{N_{L}}} X_{i}^{L} \subset \mathbb{R}^{n}$ denote the model validity sets which are assumed to contain the origin in their interiors. Note that $X_{m}$ throughout the paper will denote the state constraints, while $U_{m}$ will denote the input constraints. We do not consider explicitly constraints on the output, but note that these can be mapped to the state-space. $X_{i}^{L}$ denotes local model validity set $i$, which is the part of the statespace in which uncertainty model $i$ is valid. The local model validity sets may be overlapping. $N_{L}$ denotes the number of local uncertain models (and associated validity sets). Further, $I_{j}:=\{1, \ldots, j\}$ and $\mathscr{I}(x):=\left\{i \in I_{N_{L}} \mid x \in X_{i}^{L}\right\}$. As usual, $x \in \mathbb{R}^{n}$ denotes the states, $u \in \mathbb{R}^{m}$ denotes the inputs, and $y \in \mathbb{R}^{r}$ denotes the outputs. The initial condition is $x_{0}$.

In (Slupphaug and Foss 1999), which only treats the state-feedback case, the $\mathscr{M}_{i} \mathrm{~s}$ are given by polytopic affine difference inclusions. Here we will instead use affine affine parameter dependent (affine APD) models ${ }^{1}$ (Apkarian et al. 1996), (Gahinet et al. 1996). These two uncertainty classes are equivalent in the sense that any system described by affine APD models can equivalently be described by polytopic affine difference inclusions, and vice versa. The reason for introducing the affine APD models, is that using this model class provides a more natural way of introducing quadratic parameter dependent Lyapunov functions. Using such Lyapunov functions will reduce conservatism in the stability tests compared to the tests given in (Slupphaug and Foss 1999), where quadratic parameter independent Lyapunov functions were used.

The affine APD models are given by that $\forall i \in\left\{1, \ldots, N_{L}\right\}$

$$
\begin{gathered}
\mathscr{M}_{i}(x, u, \Theta):=\left\{\hat{x} \mid \exists \theta_{k} \in \Theta, \hat{x}=A^{i}\left(\theta_{k}\right) x+B^{i}\left(\theta_{k}\right) u+c^{i}\left(\theta_{k}\right)\right\} \\
\mathscr{H}_{i}(x, \Theta):=\left\{y \mid \exists \theta_{k} \in \Theta, y=C^{i}\left(\theta_{k}\right) x+d^{i}\left(\theta_{k}\right)\right\}
\end{gathered}
$$

where

$$
A^{i}\left(\theta_{k}\right):=A_{0}^{i}+\sum_{j=1}^{N_{\theta}} A_{j}^{i} \theta_{k, j}, B^{i}\left(\theta_{k}\right):=B_{0}^{i}+\sum_{j=1}^{N_{\theta}} B_{j}^{i} \theta_{k, j} \text { and } c^{i}\left(\theta_{k}\right):=c_{0}^{i}+\sum_{j=1}^{N_{\theta}} c_{j}^{i} \theta_{k, j}
$$

and

$$
C^{i}\left(\theta_{k}\right):=C_{0}^{i}+\sum_{j=1}^{N_{\theta}} C_{j}^{i} \theta_{k, j} \text { and } d^{i}\left(\theta_{k}\right):=d_{0}^{i}+\sum_{j=1}^{N_{\theta}} d_{j}^{i} \theta_{k, j}
$$

where $\theta_{k}=\left(\theta_{k, 1}, \ldots, \theta_{k, N_{\theta}}\right)^{T}$ is the possibly time-varying parameter vector and $N_{\theta}$ denotes the number of parameters. The parameter vector is assumed to be in a hyperrectangle (also called parameter box)

$$
\Theta:=\left\{\theta=\left(\theta_{1}, \ldots, \theta_{N_{\theta}}\right)^{T} \mid \forall j \in I_{N_{\theta}}, \theta_{j} \in[0,1]\right\}=[0,1]^{N_{\theta}} .
$$


It may seem restrictive to allow all the parameters only to be in the interval $[0,1]$ but a simple scaling argument shows that this can always be obtained (assuming, of course, that the parameter is indeed partially unknown with known upper and lower bounds). The same scaling argument implies of course that the parameters' bounds can be made equal to $\Theta$ in all the local model validity sets. Note that in the discrete-time case considered herein the parameters' rate-of-variation will be bounded whenever the parameters are, in fact they will be contained in $[-1,1]$. Thus, as opposed to the continuous-time case, using quadratic parameter dependent Lyapunov functions will be less conservative than using quadratic parameter independent ones even if explicit bounds on the parameters' rate-of-variation are not known. It should be noted that the associated rate-of-variation in real time of course will depend on the sampling period associated with the discrete-time model.

It may also seem restrictive that the $\mathscr{M}_{i} \mathrm{~s}$ and the $\mathscr{H}_{i}$ s have equivalent local model validity sets, but if they were different, one could just use the same local uncertainty models for the dynamics part and/or the output part in (appropriately chosen) different new local model validity sets.

If $0 \in$ Closure $X_{i}^{L}$, the associated $c_{j}^{i}$ s and $d_{j}^{i}$ s must be zero. This requirement means that the equilibrium control input for the state set-point is assumed to be known.

\section{Controller synthesis}

In this section, we will develop a controller for (1) that stabilizes the origin in the state space using output feedback while obeying control- and state constraints. First we treat the static output feedback case, second control constraints are considered before we show how to embed dynamic output feedback.

\subsection{Affine quadratic stabilization}

3.1.1. Piecewise affine output feedback Analogous to the state-feedback case treated in (Slupphaug and Foss 1999), we will finitely parameterize the output feedback as a piecewise affine output feedback. With the first $M_{L}^{o}$ feedback validity sets - the feedback validity sets containing the origin in their closure--we associate a linear output feedback

$$
u_{k}:=K_{j} y_{k} \quad \text { when } \quad y_{k} \in Y_{j}^{L}, j \in I_{M_{L}^{o}} .
$$

With all other feedback validity sets, we associate an affine output feedback

$$
u_{k}:=K_{j} y_{k}+k_{j} \text { when } y_{k} \in Y_{j}^{L}, j \in\left\{M_{L}^{o}+1, \ldots, M_{L}\right\} .
$$

The feedback validity sets $Y_{j}^{L}$ are assumed to partition the output space $\mathbb{R}^{r}$, meaning that the controller is indeed well defined. If one has the special case of state feedback, one can of course replace $Y_{j}^{L}$ with $X_{i}^{L}$ was done in (Slupphaug and Foss 1999).

3.1.2. Connecting output feedback to the state-space We have the local model validity sets $X_{i}^{L}, i \in I_{N_{L}}$, and the non-overlapping local feedback validity sets $Y_{j}^{L}, j \in I_{M_{L}}$, covering the state-space, $X_{m}$, and output-space, $\mathbb{R}^{r}$, respectively. Obviously, the openloop dynamics is associated with the local model validity sets, and the choice of feedback is associated with the local feedback validity sets. We will define subsets $X_{i j}$ of $X_{m}$ such that, loosely speaking, the closed-loop dynamics on $X_{i j}$ is associated with open loop dynamics $\mathscr{M}_{i}$ and feedback $j$. We will name these sets intersection sets. These subsets will exactly cover $X_{m}$ and may be overlapping. 
Formally, the intersection sets are given as

$$
X_{i j}:=X_{i}^{L} \cap\left\{x \mid \exists \theta \in \Theta, C^{i}(\theta) x+d^{i}(\theta) \in Y_{j}^{L}\right\} .
$$

The states in $X_{i j}$ are affected by the dynamics defined on $X_{i}^{L}$ and possibly, depending on $\theta$, by the feedback used in $Y_{j}^{L}$. Note that several of the intersection sets might be empty. Also, note that even if $X_{i}^{L}$ and $Y_{j}^{L}$ are polytopic, $X_{i j}$ will in general not be polytopic or even convex when output model $i$ is uncertain. This is due to the multiplication of the states and parameters. If, however, output model $i$ is certain (independent of $\theta$ ), then $X_{i j}$ will be polytopic.

The intersection sets will form the basis for using the S-procedure when deriving stability conditions for the closed-loop in Section 3.1.5.

3.1.3. Set Approximations This is done as in (Slupphaug and Foss 1999), but we repeat it here for completeness. When formulating the conditions for affine quadratic constrained stabilization, it is sensible to approximate the intersection sets and the state- and control constraints using polytopes or ellipsoids ${ }^{2}$. We index only the nonempty $X_{i j} \mathrm{~s}$ : The $X_{i j} \mathrm{~s}$ containing the origin in their closure are outer approximated by unbounded polytopes, and are indexed with $l \in I_{N^{o}}$, the $X_{i j} \mathrm{~s}$ not containing the origin in their closure are outer approximated either by possibly bounded polytopes and indexed in $\left\{N^{o}+1, \ldots, N^{p}\right\}$, or by ellipsoids and indexed in $\left\{N^{p}+1, \ldots, N\right\}$. With this indexing, for each $l$ there exists a unique pair $(i, j)$ which will be denoted $\left(i_{l}, j_{l}\right)$, thus intersection set number $l$ is $X_{i_{i j}}$. Note that $N^{o}$ denotes the number of intersection sets containing the origin in their closure, while $N^{p}$ is the number of intersection sets outer approximated by polytopes, and $N$ is the total number of non-empty intersection sets.

Thus, for $l \in I_{N^{\circ}}$ the polytope

$$
\left\{x \mid E_{l} x \leqslant 0\right\} \supset X_{i_{l} j_{l}}
$$

is used as an outer approximation of $X_{i_{i} j_{l}}$. For $l \in\left\{N^{o}+1, \ldots, N^{p}\right\}$, assume that the polytope

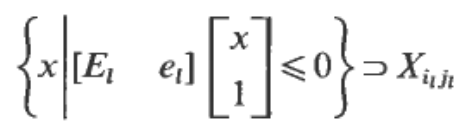

is used, and, finally, for $l \in\left\{N^{p}+1, \ldots, N\right\}$, assume that the ellipsoid

$$
\left\{x \mid\left[\begin{array}{c}
x \\
1
\end{array}\right]^{T}\left[\begin{array}{ll}
E_{l} & e_{l} \\
e_{l}^{T} & \varepsilon_{l}
\end{array}\right]\left[\begin{array}{l}
x \\
1
\end{array}\right] \leqslant 0\right\} \supset X_{i_{i} j_{t}}
$$

is used.

Furthermore, assume that the state-space model validity- and constraint set $X_{m}$ is inner approximated as follows ${ }^{3}$

$$
0 \in \bigcap_{i \in I_{N_{q x}}}\left\{x \mid\left\|x-x_{i, c}\right\|_{H_{i, x}}^{2} \leqslant 1\right\} \subset X_{m},
$$

i.e. by an intersection of ellipsoids where $x_{i, c}$ denotes the centers of the ellipsoids, and $N_{q x}$ denotes the number of ellipsoids.

Similarly, we assume

$$
0 \in \bigcap_{i \in I_{N_{q u}}}\left\{u \mid\left\|u-u_{i, c}\right\|_{H_{i, u}}^{2} \leqslant 1\right\} \subset U_{m},
$$


where $U_{m}$ is the control model validity- and constraint set. Note that any of the outer approximations (6), (7), and (8), exists for any set, and that they are the natural outer approximations to choose when formulating LMI problems for piecewise linear (and affine) systems (Hassibi and Boyd 1998), (Johansson 1999).

The inner approximations (9) and (10) also exist for any $X_{m}$ and $U_{m}$ with the origin in the interior, and the origin can and should be placed in the interior of each of the intersections.

3.1.4. Stability definition We will use the following stability notion, see e.g. (Slupphaug and Foss 1999) and (Scherer and Weiland 1999) for similar definitions.

\section{Definition 3.1 (Affine quadratic stability)}

Given the system

$$
x_{k+1}=A\left(\theta_{k}, x_{k}\right) x_{k}+c\left(\theta_{k}, x_{k}\right),
$$

where $k \in \mathbb{N}, x_{k} \in X_{m} \subset \mathbb{R}^{n}, x_{0}$ given and $c\left(\theta_{k}, 0\right)=0$ for all $\theta_{k} \in \Theta \subset \mathbb{R}^{N_{\theta}}$. Define the (affine) Lyapunov matrix function $P\left(\theta_{k}\right)=P_{0}+\Sigma_{j=1}^{N_{1}} \theta_{k, j} P_{j}$. The origin is an affinely quadratically stable equilibrium for the system (11) if there exists $\varepsilon>0$ and symmetric matrices $M>0, P_{0}, P_{1}, \ldots, P_{N_{\theta}}$ and $N(\varepsilon)^{4} \subset X_{m}$ such that for all $x_{k} \in N(\varepsilon), \theta_{k}, \theta_{k+1} \in \Theta$

$$
\begin{gathered}
\left(A\left(\theta_{k}, x_{k}\right) x_{k}+c\left(\theta_{k}, x_{k}\right)\right)^{T} P\left(\theta_{k+1}\right)\left(A\left(\theta_{k}, x_{k}\right) x_{k}+c\left(\theta_{k, x_{k}}\right)\right)-x_{k}^{T} P\left(\theta_{k}\right) x_{k} \leqslant-x_{k}^{T} M x_{k} \\
P\left(\theta_{k}\right)>0 .
\end{gathered}
$$

If, in addition, there exist scalars $\alpha_{0}, \alpha_{1}, \ldots, \alpha_{N_{\theta}}$ rendering the affine function $\alpha(\theta):=\alpha_{0}+\Sigma_{j}^{N_{\underline{\theta}}} \theta_{j} \alpha_{j}>0$ for all $\theta \in \Theta$ such that for a given set $\tilde{R}_{A}$,

$$
\tilde{R}_{A} \subset\left\{x \mid \exists \theta \in \Theta, x^{T} P(\theta) x \leqslant \alpha(\theta)\right\} \subset N(\varepsilon),
$$

then the origin is said to be an affinely quadratically stable equilibrium for the system (11) with a region of attraction associated with $\tilde{R}_{A}$ of at least $\{x \mid \exists \theta \in \Theta$, $\left.x^{T} P(\theta) x \leqslant \alpha(\theta)\right\}$.

3.1.5. Synthesis: Robust output feedback Next we derive LMIs subject to a spectral radius constraint on the product of two positive definite matrices (a specially structured BMI) for affine quadratic stabilization of the origin of the closed loop (1) with (4), (5). This extends the results in (Slupphaug and Foss 1999) to the output feedback case. In addition we use a parameter dependent Lyapunov function making it possible to take into account the implicit bounds on the parameters' rate-of-variation that are present in this discrete-time case (cf. the discussion in Section 2). This will make the current approach less conservative in the state-feedback case than (Slupphaug and Foss 1999) where a parameter independent Lyapunov function was used.

In the following result we will need the affine functions:

$$
\begin{gathered}
W^{l}(\theta):=W_{o}^{l}+\sum_{j=1}^{N_{\theta}} \theta_{j} W_{j}^{l} \\
\tau^{l}(\theta):=\tau_{o}^{l}+\sum_{j=1}^{N_{\theta}} \theta_{j} \tau_{j}^{l}
\end{gathered}
$$




$$
\begin{array}{r}
\beta(\theta):=\beta_{o}+\sum_{j=1}^{N_{\theta}} \theta_{j} \beta_{j} \\
\mu^{l}(\theta):=\mu_{o}^{l}+\sum_{j=1}^{N_{\theta}} \theta_{j} \mu_{j}^{l}
\end{array}
$$

The $W_{j}^{l} \mathrm{~s}$ are symmetric matrices whose dimension are the row dimension of the corresponding $E_{l} \mathrm{~s}$, denoted $n_{E_{l}}$. The $\tau_{j}^{l} \mathrm{~s}, \beta_{j} \mathrm{~s}$ and $\mu_{j}^{l} \mathrm{~s}$ are scalars.

Theorem 3.1 Let $\mathscr{F}_{i}$ index those parameters in local model $i$ that enters quadratically in (12), (14) and (16), and let $\Theta_{0}$ be the corners of the parameter box $\Theta$, i.e. $\Theta_{0}=\{0,1\}^{N_{\theta}}$. Then, if $\exists M>0, T_{t}^{l} \geqslant 0$ for $\left\{(l, t) \mid l \in I_{N}, t \in \mathscr{J}_{i}\right\}$, symmetric matrices

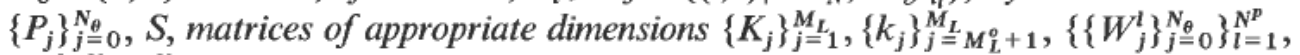
$\left\{\left\{\tau_{j}^{l}\right\}_{j}^{N_{\underline{\theta}}}\right\}_{l=N^{p}+1}^{N} \forall l \in I_{N^{o}}, \theta \in \Theta_{0}$

$$
\begin{gathered}
{\left[\begin{array}{cc}
S & A^{i_{l}}(\theta)+B^{i_{l}}(\theta) K_{j_{l}} C^{i_{1}}(\theta) \\
* & P(\theta)-M-E_{l}^{T} W^{l}(\theta) E_{l}
\end{array}\right]-\sum_{t \in \mathscr{I}_{i_{l}}} T_{t}^{l} \theta_{t}^{2} \geqslant 0} \\
{\left[\begin{array}{cc}
0 & B^{i_{l}} K_{j_{l}} C_{t}^{i_{l}} \\
* & 0
\end{array}\right]-T_{t}^{l} \leqslant 0 \forall t \in \mathscr{I}_{i_{l}}}
\end{gathered}
$$

$\forall l \in\left\{N^{o}+1, \ldots, N^{p}\right\}, \theta \in \Theta_{0}$

$$
\begin{gathered}
{\left[\begin{array}{ccc}
S & A^{i_{l}}(\theta)+B^{i_{1}}(\theta) K_{j_{l}} C^{i_{1}}(\theta) & B^{i_{1}}(\theta) K_{j_{l}} d^{i_{l}}(\theta)+B^{i_{l}}(\theta) k_{j_{l}}+c^{i_{l}}(\theta) \\
* & P(\theta)-M-E_{l}^{T} W^{l}(\theta) E_{l} & -E_{l}^{T} W^{l}(\theta) e_{l} \\
* & * & -e_{l}^{T} W^{l}(\theta) e_{l}
\end{array}\right]-\sum_{t \in \mathscr{g}_{i_{l}}} T_{t}^{l} \theta_{t}^{2} \geqslant 0} \\
{\left[\begin{array}{ccc}
0 & B_{t}^{i_{l}} K_{j_{l}} C_{t}^{i_{l}} & B_{t}^{i_{l}} K_{j_{l}} d_{l}^{i_{l}} \\
* & 0 & 0 \\
* & * & 0
\end{array}\right]-T_{t}^{l} \leqslant 0 \forall t \in \mathscr{I}_{i_{l}}}
\end{gathered}
$$

$\forall l \in\left\{N^{o}+1, \ldots, N\right\}, \theta \in \Theta_{0}$

$$
\begin{aligned}
& {\left[\begin{array}{ccc}
S & A^{i_{i}}(\theta)+B^{i_{1}}(\theta) K_{j_{l}} C^{i_{l}}(\theta) & B^{i_{l}}(\theta) K_{j_{l}} d^{i_{l}}(\theta)+B^{i_{l}}(\theta) k_{j_{l}}+c^{i_{l}}(\theta) \\
* & P(\theta)-M+\tau^{l}(\theta) E_{l} & \tau^{l}(\theta) e_{l} \\
* & * & \tau^{l}(\theta) \varepsilon_{l}
\end{array}\right]-\sum_{t \in \mathcal{g}_{i_{l}}} T_{t}^{l} \theta_{l}^{2} \geqslant 0} \\
& {\left[\begin{array}{ccc}
0 & B_{l}^{i_{l}} K_{j_{l}} C_{t}^{i_{l}} & B_{t}^{i_{l}} K_{j_{l}} d_{t}^{i_{l}} \\
* & 0 & 0 \\
* & * & 0
\end{array}\right]-T_{t}^{l} \leqslant 0 \forall t \in \mathscr{J}_{i_{l}}}
\end{aligned}
$$

$\forall \theta \in \Theta_{0}$

$$
0<P(\theta) \leqslant S^{-1}
$$

and $^{5} \forall l \in I_{N^{p}}, \theta \in \Theta_{0}$ 


$$
W^{l}(\theta) \in \mathbb{R}_{+}^{n_{E_{1}} \times n_{E_{l}}},
$$

then the origin is an affinely quadratically stable equilibrium for the closed-loop system. If, in addition, there exist reals $\left\{\alpha_{j}\right\}_{j}^{N_{\theta}}{ }_{0}$ and $\left\{\beta_{j}\right\}_{j=0}^{N_{0}}$ such that $\forall \theta \in \Theta_{0}$

$$
\left[\begin{array}{cc}
P(\theta)-\beta(\theta) R_{A} & 0 \\
0 & \beta(\theta)-\alpha(\theta)
\end{array}\right] \leqslant 0
$$

and reals $\left\{\left\{\mu_{j}^{l}\right\}_{j}^{N_{\theta}}\right\}_{l \in I_{N_{q x}}}$ such that $\forall l \in I_{N_{q x}}, \theta \in \Theta_{0}$

$$
\left[\begin{array}{cc}
\mu^{l}(\theta) H_{l, x}-P(\theta) & -\mu^{l}(\theta) H_{l, x} x_{l, c} \\
* & \mu^{l}(\theta)\left(x_{l, c}^{T} H_{l, x} x_{l, c}-1\right)+\alpha(\theta)
\end{array}\right] \leqslant 0,
$$

then the origin is an affinely quadratically stable equilibrium for the closed-loop system with a region of attraction associated with $\left\{x \mid\|x\|_{R_{A}}^{2} \leqslant 1\right\}$ of at least $x \mid \exists \theta \in \Theta$, $\left.x^{T} P(\theta) x \leqslant \alpha(\theta)\right\}$.

All * are to be induced by symmetry. Now, the proof of this Theorem is similar to and along the same lines as the proof of Theorem 1 in (Slupphaug and Foss 1999), the main differences being that we now incorporate uncertain output feedback and hence need arguments that are based on multi-convexity and the results of (Gahinet at al. 1996). Also, the S-procedure variables are now parameter dependent.

Proof. By Definition 3.1, the origin is an affinely quadratically stable equilibrium for the closed loop if $\exists M>0,\left\{P_{j}\right\}_{j=0}^{N_{\underline{\theta}}},\left\{K_{j}\right\}_{j \pm 1}^{M_{L}},\left\{k_{j}\right\}_{j=M_{L}^{o+1}}^{M_{L}}, \forall l \in I_{N^{0}}, \quad x_{k} \in X_{i_{i} j_{l}}, \theta_{k}$, $\theta_{k+1} \in \Theta$

$$
\begin{aligned}
& \left\{\left(A^{i_{1}}\left(\theta_{k}\right)+B^{i_{l}}\left(\theta_{k}\right) K_{j_{l}} C^{i_{1}}\left(\theta_{k}\right)\right) x_{k}\right\}^{T} P\left(\theta_{k+1}\right) *-x_{k}^{T} P\left(\theta_{k}\right) x_{k} \leqslant-x_{k}^{T} M x_{k} \\
& \forall l \in\left\{N^{o}+1, \ldots, N\right\}, x_{k} \in X_{i_{i} j_{l}}, \theta_{k}, \theta_{k+1} \in \Theta \\
& \left\{\left[A^{i_{1}}\left(\theta_{k}\right)+B^{i_{1}}\left(\theta_{k}\right) K_{j_{l}} C^{i_{1}}\left(\theta_{k}\right) B^{i_{l}}\left(\theta_{k}\right) K_{j_{l}} d^{i_{1}}\left(\theta_{k}\right)+B^{i_{1}}\left(\theta_{k}\right) k_{j_{l}}+c^{i_{l}}\left(\theta_{k}\right)\right]\left[\begin{array}{c}
x_{k} \\
1
\end{array}\right]\right\}^{T} P\left(\theta_{k+1}\right) * \\
& -\left[\begin{array}{c}
x_{k} \\
1
\end{array}\right]^{T}\left[\begin{array}{cc}
P\left(\theta_{k}\right) & 0 \\
0 & 0
\end{array}\right]\left[\begin{array}{c}
x_{k} \\
1
\end{array}\right] \leqslant-\left[\begin{array}{c}
x_{k} \\
1
\end{array}\right]^{T}\left[\begin{array}{cc}
M & 0 \\
0 & 0
\end{array}\right]\left[\begin{array}{c}
x_{k} \\
1
\end{array}\right]
\end{aligned}
$$

and $\forall \theta_{k} \in \Theta$

$$
P\left(\theta_{k}\right)>0 .
$$

Next, by using the S-procedure to cover the different intersection sets (or, $X_{i j} \mathrm{~s}$ ) (see (Slupphaug 1998) for details, we here extend the S-procedure in a natural way by using parameter dependent S-procedure variables), we get that (22)-(24) are implied by $\exists M>0,\left\{P_{j}\right\}_{j=0}^{N_{\underline{\theta}}},\left\{K_{j}\right\}_{j=1}^{M_{L_{1}}},\left\{k_{j}\right\}_{j}^{M_{\underline{L}} M_{L+1}^{o}},\left\{\left\{W_{j}^{l}\right\}_{j}^{N_{\underline{\underline{\theta}}}}\right\}_{l=1}^{N^{p}},\left\{\left\{\tau_{j}^{l}\right\}_{j}^{N_{\underline{\theta}}}\right\}_{l=N^{p}+1}^{N} \forall l \in I_{N^{o}}$, $a \in \mathbb{R}^{n}, \theta_{k}, \theta_{k+1} \in \Theta$

$$
\begin{aligned}
& \left\{\left(A^{i_{l}}\left(\theta_{k}\right)+B^{i_{1}}\left(\theta_{k}\right) K_{j_{l}} C^{i_{l}}\left(\theta_{k}\right)\right) a\right\}^{T} P\left(\theta_{k+1}\right) *-a^{T} P\left(\theta_{k}\right) a+a^{T} M a+a^{T} E_{l}^{T} W^{l}\left(\theta_{k}\right) E_{l} a \leqslant 0 \\
& \forall l \in\left\{N^{o}+1, \ldots, N^{p}\right\}, a \in \mathbb{R}^{n}, \theta_{k}, \theta_{k+1} \in \Theta
\end{aligned}
$$




$$
\begin{aligned}
& \left\{\left[A^{i_{l}}\left(\theta_{k}\right)+B^{i_{l}}\left(\theta_{k}\right) K_{j_{l}} C^{i_{l}}\left(\theta_{k}\right) B^{i_{l}}\left(\theta_{k}\right) K_{j_{l}} d^{i_{i}}\left(\theta_{k}\right)+B^{i_{l}}\left(\theta_{k}\right) k_{j_{l}}+c^{i_{l}}\left(\theta_{k}\right)\right]\left[\begin{array}{l}
a \\
1
\end{array}\right]\right\}^{T} P\left(\theta_{k+1}\right) * \\
& -\left[\begin{array}{l}
a \\
1
\end{array}\right]^{T}\left[\begin{array}{cc}
P\left(\theta_{k}\right) & 0 \\
0 & 0
\end{array}\right]\left[\begin{array}{l}
a \\
1
\end{array}\right]+\left[\begin{array}{c}
a \\
1
\end{array}\right]^{T}\left[\begin{array}{cc}
M & 0 \\
0 & 0
\end{array}\right]\left[\begin{array}{l}
a \\
1
\end{array}\right] \\
& +\left[\begin{array}{c}
a \\
1
\end{array}\right]^{T}\left[\begin{array}{c}
E_{l}^{T} \\
e_{l}^{T}
\end{array}\right] W^{l}\left(\theta_{k}\right)\left[\begin{array}{ll}
E_{l} & e_{l}
\end{array}\right]\left[\begin{array}{l}
a \\
1
\end{array}\right] \leqslant 0
\end{aligned}
$$

and $\forall l \in\left\{N^{p}+1, \ldots, N\right\}, a \in \mathbb{R}^{n}, \theta_{k}, \theta_{k+1} \in \Theta$

$$
\begin{aligned}
& \left\{\left[A^{i_{1}}\left(\theta_{k}\right)+B^{i_{l}}\left(\theta_{k}\right) K_{j_{l}} C^{i_{l}}\left(\theta_{k}\right) B^{i_{1}}\left(\theta_{k}\right) K_{j_{l}} d^{i_{l}}\left(\theta_{k}\right)+B^{i_{l}}\left(\theta_{k}\right) k_{j_{l}}+c^{i_{l}}\left(\theta_{k}\right)\right]\left[\begin{array}{l}
a \\
1
\end{array}\right]\right\}^{T} P\left(\theta_{k+1}\right) * \\
& -\left[\begin{array}{c}
a \\
1
\end{array}\right]^{T}\left[\begin{array}{cc}
P\left(\theta_{k}\right) & 0 \\
0 & 0
\end{array}\right]\left[\begin{array}{l}
a \\
1
\end{array}\right]+\left[\begin{array}{c}
a \\
1
\end{array}\right]^{T}\left[\begin{array}{cc}
M & 0 \\
0 & 0
\end{array}\right]\left[\begin{array}{l}
a \\
1
\end{array}\right] \\
& -\tau^{l}\left(\theta_{k}\right)\left[\begin{array}{c}
a \\
1
\end{array}\right]^{T}\left[\begin{array}{ll}
E_{l} & e_{l} \\
e_{l} & \varepsilon_{l}
\end{array}\right]\left[\begin{array}{l}
a \\
1
\end{array}\right] \leqslant 0
\end{aligned}
$$

$\forall \theta_{k} \in \Theta$

$$
P\left(\theta_{k}\right)>0
$$

$\forall l \in I_{N^{p}}, \theta_{k} \in \Theta$

$$
W^{\prime}\left(\theta_{k}\right) \in \mathbb{R}_{E_{i}}^{n_{1}} \times n_{E_{i}}
$$

and $\forall l \in\left\{N^{p}+1, \ldots, N\right\}, \theta_{k} \in \Theta$

$$
\tau^{l}\left(\theta_{k}\right) \geqslant 0 .
$$

Using the fact that $\left[\begin{array}{ll}z^{T} & 1\end{array}\right] Q\left[\begin{array}{ll}z^{T} & 1\end{array}\right]^{T} \geqslant 0 \forall z \Leftrightarrow Q \geqslant 0$, this is equivalent to $\exists M>0$, $\left\{P_{j}\right\}_{j}^{N_{\underline{\theta}}},\left\{K_{j}\right\}_{j=1}^{M_{L}},\left\{k_{j}\right\}_{j=M_{L}^{M_{L}}}^{M_{L}},\left\{\left\{W_{j}^{l}\right\}_{j=0}^{N_{\underline{\theta}}}\right\}_{l=1}^{N^{p}},\left\{\left\{\tau_{j}^{l}\right\}_{j=0}^{N_{\theta}}\right\}_{l=N^{p}+1}^{N} \forall l \in I_{N^{o}}, \theta_{k}, \theta_{k+1} \in \Theta$

$$
\left(A^{i_{i}}\left(\theta_{k}\right)+B^{i_{l}}\left(\theta_{k}\right) K_{j_{l}} C^{i_{i}}\left(\theta_{k}\right)\right)^{T} P\left(\theta_{k+1}\right) *-P\left(\theta_{k}\right)+M+E_{l}^{T} W^{l}\left(\theta_{k}\right) E_{l} \leqslant 0
$$

$\forall l \in\left\{N^{o}+1, \ldots, N^{p}\right\}, \theta_{k}, \theta_{k+1} \in \Theta$

$$
\begin{aligned}
& {\left[A^{i_{1}}\left(\theta_{k}\right)+B^{i_{I}}\left(\theta_{k}\right) K_{j_{l}} C^{i_{l}}\left(\theta_{k}\right) \quad B^{i_{1}}\left(\theta_{k}\right) K_{j_{l}} d^{i_{1}}\left(\theta_{k}\right)+B^{i_{l}}\left(\theta_{k}\right) k_{j_{l}}+c^{i_{l}}\left(\theta_{k}\right)\right]^{T} P\left(\theta_{k+1}\right) *} \\
& -\left[\begin{array}{cc}
P\left(\theta_{k}\right) & 0 \\
0 & 0
\end{array}\right]+\left[\begin{array}{cc}
M & 0 \\
0 & 0
\end{array}\right]+\left[\begin{array}{c}
E_{l}^{T} \\
e_{l}^{T}
\end{array}\right] W^{l}\left(\theta_{k}\right)\left[\begin{array}{ll}
E_{l} & e_{l}
\end{array}\right] \leqslant 0
\end{aligned}
$$

and $\forall l \in\left\{N^{p}+1, \ldots, N\right\}, \theta_{k}, \theta_{k+1} \in \Theta$

$$
\begin{aligned}
& {\left[A^{i_{l}}\left(\theta_{k}\right)+B^{i_{l}}\left(\theta_{k}\right) K_{j_{l}} C^{i_{l}}\left(\theta_{k}\right) B^{i^{i}}\left(\theta_{k}\right) K_{j_{l}} d^{i_{l}}\left(\theta_{k}\right)+B^{i_{l}}\left(\theta_{k}\right) k_{j_{l}}+c^{i_{l}}\left(\theta_{k}\right)\right]^{T} P\left(\theta_{k+1}\right) *} \\
& -\left[\begin{array}{cc}
P\left(\theta_{k}\right) & 0 \\
0 & 0
\end{array}\right]+\left[\begin{array}{cc}
M & 0 \\
0 & 0
\end{array}\right]-\tau^{l}\left(\theta_{k}\right)\left[\begin{array}{cc}
E_{l} & e_{l} \\
e_{l} & \varepsilon_{l}
\end{array}\right] \leqslant 0
\end{aligned}
$$


and $\forall \theta_{k} \in \Theta$

$$
P\left(\theta_{k}\right)>0
$$

$\forall l \in I_{N^{p}}, \theta_{k} \in \Theta$

$$
W^{l}\left(\theta_{k}\right) \in \mathbb{R}_{f}^{n_{E_{i}}} \times n_{E_{i}}
$$

and $\forall l \in\left\{N^{p}+1, \ldots, N\right\}, \theta_{k} \in \Theta$

$$
\tau^{l}\left(\theta_{k}\right) \geqslant 0
$$

By applying Schur complements on equations (25)-(27) (note that $\tau^{l}\left(\theta_{k}\right)>0$ necessarily since $P\left(\theta_{k}\right)>0$ and $\varepsilon_{l}>0$ ), introducing a new symmetric matrix variable $S$, and noting that (see Corollary 7.7.4 in (Horn and Johnson 1992)) $0<S \leqslant P\left(\theta_{k+1}\right)^{-1} \Leftrightarrow 0<P\left(\theta_{k+1}\right) \leqslant S^{-1}$, this is equivalent to $\exists M>0,\left\{P_{j}\right\}_{j}^{N_{\underline{\theta}}}, S$, $\left\{K_{j}\right\}_{j=1}^{M_{L}},\left\{k_{j}\right\}_{j}^{M_{L}} M_{M_{L}^{o}+1},\left\{\left\{W_{j}^{l}\right\}_{j=0}^{N_{0}}\right\}_{l=1}^{N^{p}},\left\{\left\{\tau_{j}^{l}\right\}_{j}^{N_{\underline{\theta}}}\right\}_{l=N^{p}+1}^{N} \forall l \in I_{N^{o}}, \theta \in \Theta^{6}$

$$
\left[\begin{array}{ll}
S & A^{i_{l}}(\theta)+B^{i_{l}}(\theta) K_{j_{l}} C^{i_{l}}(\theta) \\
* & P(\theta)-M-E_{l}^{T} W^{l}(\theta) E_{l}
\end{array}\right] \geqslant 0
$$

$\forall l \in\left\{N^{o}+1, \ldots, N^{p}\right\}, \theta \in \Theta$

$$
\left[\begin{array}{ccc}
S & A^{i_{l}}(\theta)+B^{i_{l}}(\theta) K_{j_{l}} C^{i_{l}}(\theta) & B^{i_{l}}(\theta) K_{j_{l}} d^{i_{l}}(\theta)+B^{i_{l}}(\theta) k_{j_{l}}+c^{i_{l}}(\theta) \\
* & P(\theta)-M-E_{l}^{T} W^{l}(\theta) E_{l} & -E_{l}^{T} W^{l}(\theta) e_{l} \\
* & * & -e_{l}^{T} W^{l}(\theta) e_{l}
\end{array}\right] \geqslant 0
$$

$\forall l \in\left\{N^{o}+1, \ldots, N\right\}, \theta \in \Theta$

$$
\left[\begin{array}{ccc}
S & A^{i_{1}}(\theta)+B^{i_{l}}(\theta) K_{j_{l}} C^{i_{l}}(\theta) & B^{i_{l}}(\theta) K_{j_{l}} d^{i_{l}}(\theta)+B^{i_{l}}(\theta) k_{j_{l}}+c^{i_{l}}(\theta) \\
* & P(\theta)-M+\tau^{l}(\theta) E_{l} & \tau^{l}(\theta) e_{l} \\
* & * & \tau^{l}(\theta) \varepsilon_{l}
\end{array}\right] \geqslant 0
$$

$\forall \theta \in \Theta$

$$
0<P(\theta) \leqslant S^{-1}
$$

and that $\forall l \in I_{N^{p}}, \theta \in \Theta$

$$
W^{\prime}(\theta) \in \mathbb{R}_{F_{t}}^{n_{E_{t}}} \times n_{E_{i}}
$$

Now, all these matrix inequalities are parametrized in the continuous set $\Theta$. To get a finite number of matrix inequalities, we find conditions that allow us to replace $\Theta$ with the finite set $\Theta_{0}$. One such condition is that the matrix inequalities are multiconvex $^{7}$ in the parameters (Gahinet et al. 1996, Lemma 3.1), (Apkarian and Tuan $2000 a$ ). Multi-convex essentially means 'convex in each of the parameters' in our case, where $\Theta$ is a hyper-rectangle.

If the matrix inequalities are affine in the parameters, then they are always multiconvex, meaning that we can replace $\Theta$ with $\Theta_{0}$ without conservatism. If, as (possibly) in our case, some of the matrix inequalities are quadratic in the parameters, they can be written as (when keeping all parameters constant, except one particular $\theta_{t}$ that enters quadratically)

$$
X+Y \theta_{t}+Z \theta_{t}^{2} .
$$


Convexity with respect to this parameter is ensured when $Z \geqslant 0$ (Gahinet at al. 1996), and this can be added as an extra LMI constraint. This might of course introduce conservatism.

In some cases, this constraint can be difficult (in our case, in fact impossible except in the trivial case) to satisfy. We can get a less conservative constraint by instead of requiring that $Z+Y \theta_{t}+Z \theta_{t}^{2} \leqslant 0$, require that the overbound $X+Y \theta_{t}+$ $\left(Z+T_{t}\right) \theta_{t}^{2} \leqslant 0$, for some $T_{t} \geqslant 0$. The constraint then becomes $Z+T_{t} \geqslant 0$. This idea is from (Gahinet at al. 1996).

The $\theta_{t}$ s that enter non-trivially in both $B^{i}(\theta)$ and $C^{i}(\theta)$, or both $B^{i}(\theta)$ and $d^{i}(\theta)$, for some $i$ will appear quadratically in (28)-(30). For these, we must add an extra LMI constraint as explained above. Let these $\theta_{t} \mathrm{~s}$ in local model $i$ be indexed in $\mathscr{Z}_{i}$. Thus, the inequalities (12)-(19) in the theorem implies (28)-(32), and the first part of the theorem follows. The last part follows from (Slupphaug and Foss 1999) and a similar argument (based on affinity in the parameters and multi-convexity) as above.

3.1.6. Discussion We will comment on some issues regarding the above theorem:

- Compared to the result for the state-feedback case in (Slupphaug and Foss 1999) the quadratic (in $\theta)$ uncertain terms $B^{i_{1}}(\theta) K_{j_{l}} C^{i_{l}}(\theta)$ and $B^{i_{l}}(\theta) K_{j_{l}} d^{i_{l}}(\theta)$ give rise to a possibly large set of additional LMI conditions (and extra LMI variables): (13), (15) and (17).

- The symmetric matrices $T_{t}^{l}$ will possibly introduce a great number of scalar LMI variables, depending on the size of the $\mathscr{H}_{i} \mathrm{~s}$. This problem can be reduced by instead letting $T_{t}^{l}=\sigma_{t}^{l} I$, where the $\sigma_{t}^{l}$ s are scalars. Of course, this will generally be more conservative.

- If there are no quadratic parameter terms in equations (12), (14) and (16) (that is, $\mathscr{J}_{i}, i \in I_{N_{L}}$ is empty), the extra LMI conditions ((13), (15) and (17)) which introduce conservatism, vanish. This is the case when the same parameters do not appear in both $C^{i}(\theta)$ and $B^{i}(\theta)$ and in both $d^{i}(\theta)$ and $B^{i}(\theta)$. Special cases where the quadratic terms disappear is when the input model is piecewise affine and known, i.e. the $B^{i}(\theta)=B^{i}$, or the output model is piecewise affine and known, i.e. the $C^{i}(\theta)=C^{i}$. A special case of the latter is state-feedback implying that $C^{i}=I$.

- Even though we do not use explicit information on the parameters' rate-ofvariation in the theorem, due to the implicit sampling and the bounds on $\theta$, we still implicitly take into account that the rate-of-variation is bounded (we know that $\theta_{k+1}-\theta_{k} \in[-1,1]^{N_{\theta}}$ ). This is as opposed to the continuous time case (Gahinet at al. 1996), where no information on the parameters' rate-of-variation forces $P_{1}=\ldots=P_{N_{\theta}}=0$.

- In addition to a parameter dependent Lyapunov function, other variables are also chosen to depend affinely on the parameters. This reduces conservatism compared to the case where these are not parameter dependent, but of course one has to pay by having more LMI-variables.

\subsection{Handling control input constraints}

It is possible to handle control input constraints in Theorem 3.1 by adding a number of LMI constraints. The approach we use here is the same as in (Slupphaug and Foss 1999), but we will adjust it to fit the new model uncertainty class and output feedback. 
We have to satisfy the control constraints, $U_{m}$, in all the $X_{i j} \mathrm{~s}$. For this to be the case, it is sufficient that $\forall(p, l) \in I_{N_{q u}} \times I_{N}, \theta \in \Theta$

$$
\left\|K_{j_{l}}\left(C^{i_{1}}(\theta) x+d^{i_{i}}(\theta)\right)+k_{j_{l}}-u_{p, c}\right\|_{H_{p, u}}^{2} \leqslant 1, x \in X_{i_{l} j_{l}} .
$$

Note that this condition is not necessary because of the inner approximation of $U_{m}$.

When outer approximating the $X_{i j}$ s for formulating LMI conditions for satisfying control input constraints in this manner, it is only sensible to use ellipsoids, see (Slupphaug and Foss 1999). Thus, we assume that ${ }^{8}$

$$
\left\{x \mid\left[\begin{array}{c}
x \\
1
\end{array}\right]^{T}\left[\begin{array}{cc}
\tilde{E}_{l} & \tilde{e}_{l} \\
\tilde{e}_{l}^{T} & \tilde{\varepsilon}_{l}
\end{array}\right]\left[\begin{array}{c}
x \\
1
\end{array}\right] \leqslant 0\right\} \supset X_{i_{l} j_{l} .}
$$

Using the S-procedure and Schur complements on (33), we get that the LMI conditions: $\forall(p, l) \in I_{N_{q u}} \times I_{N}, \theta \in \Theta_{0} \exists\left\{\tau_{l, p, j}^{E}\right\}_{j}^{N_{\underline{\theta}}} \underline{0}_{0}$

$$
\begin{array}{ccc}
{\left[\begin{array}{ccc}
\tau_{l, p}^{E}(\theta) \tilde{E}_{l} & \tau_{l, p}^{E}(\theta) \tilde{e}_{l} & \left(K_{j_{l}} C^{i_{i}}(\theta)\right)^{T} \\
* & 1+\tau_{l, p}^{E}(\theta) \tilde{\varepsilon}_{l} & \left(k_{j_{l}}+K_{j_{l}} d^{i_{l}}(\theta)-u_{p, c}\right)^{T} \\
* & * & H_{p, u}^{-1}
\end{array}\right] \geqslant 0} \\
\multirow{2}{*}{\tau_{l, p}^{E}(\theta):=\tau_{l, p, 0}^{E}+\sum_{j=1}^{N_{\theta}} \theta_{j} \tau_{l, p, j}^{E} \geqslant 0}
\end{array}
$$

imply the sufficient condition (33) for satisfying control input constraints. Since these LMIs are affine in $\theta$, we can indeed exchange $\Theta$ with $\Theta_{0}$. From this, we get:

Theorem 3.2 If the hypotheses (12) (21) of Theorem 3.1 as well as the LMIs (34)-(35) are satisfied, then the origin is an affinely quadratically stable equilibrium for the closed loop with a region of attraction associated with $\left\{x \mid\|x\|_{R_{1}}^{2} \leqslant 1\right\}$ of at least $\left\{x \mid \exists \theta \in \Theta, x^{T} P(\theta) x \leqslant \alpha(\theta)\right\}$, and the control input constraints, $U_{m}$, are satisfied on all closed loop trajectories within $X_{m}$.

\subsection{Dynamic output feedback}

The problem of finding a (linear) dynamic output feedback controller can be transformed into the problem of finding a static output feedback controller by a system augmentation technique (see e.g. El Ghaoui et al. 1997). In our case, we want a piecewise affine dynamic output feedback controller, and the same system augmentation technique can be used.

Parameterize the dynamic output feedback controller in feedback validity set $Y_{j}^{L}, j \in I_{M_{L}}$ as

$$
\begin{array}{r}
z_{k+1}=\hat{A}_{j} z_{k}+\hat{B}_{j} y_{k}+\hat{c}_{j} \\
u_{k}=\hat{C}_{j} z_{k}+\hat{D}_{j} y_{k}+\hat{d}_{j},
\end{array}
$$

where $z_{k} \in \mathbb{R}^{n_{c}}$ and $\hat{A}_{j}, \hat{B}_{j}, \ldots, \hat{d}_{j}$ are matrices of appropriate dimensions (of course, $\hat{c}_{j}$ and $\hat{d}_{j}$ are zero in the first $M_{L}^{0}$ feedback validity sets). The controller order is denoted $n_{c}$. Note that we can choose $n_{\mathrm{c}}<n$, thus this dynamic controller design could be called a reduced- (or fixed-) order controller design. 
Now, define

$$
\hat{K}_{j}:=\left[\begin{array}{ll}
\hat{D}_{j} & \hat{C}_{j} \\
\hat{B}_{j} & \hat{A}_{j}
\end{array}\right], \hat{k}_{j}:=\left[\begin{array}{l}
\hat{d}_{j} \\
\hat{c}_{j}
\end{array}\right]
$$

The dynamics in model validity set $X_{i}^{L}$,

$$
\begin{aligned}
x_{k+1} & =A^{i}\left(\theta_{k}\right) x_{k}+B^{i}\left(\theta_{k}\right) u_{k}+c^{i}\left(\theta_{k}\right) \\
y_{k} & =C^{i}\left(\theta_{k}\right) x_{k}+d^{i}\left(\theta_{k}\right)
\end{aligned}
$$

can then be augmented with the controller dynamics in feedback validity set $Y_{j}^{L}$ as follows:

$$
\begin{gathered}
{\left[\begin{array}{c}
x_{k+1} \\
z_{k+1}
\end{array}\right]=\left[\begin{array}{cc}
A^{i}\left(\theta_{k}\right) & 0 \\
0 & 0
\end{array}\right]\left[\begin{array}{l}
x_{k} \\
z_{k}
\end{array}\right]+\left[\begin{array}{cc}
B^{i}\left(\theta_{k}\right) & 0 \\
0 & I
\end{array}\right] \hat{u}_{k}+\left[\begin{array}{c}
c^{i}\left(\theta_{k}\right) \\
0
\end{array}\right]} \\
\hat{y}_{k}=\left[\begin{array}{cc}
C^{i}\left(\theta_{k}\right) & 0 \\
0 & I
\end{array}\right]\left[\begin{array}{c}
x_{k} \\
z_{k}
\end{array}\right]+\left[\begin{array}{c}
d^{i}\left(\theta_{k}\right) \\
0
\end{array}\right]
\end{gathered}
$$

with the feedback

$$
\hat{u}_{k}=\hat{K}_{j} \hat{y}_{k}+\hat{k}_{j}
$$

Note that

$$
\hat{u}_{k}=\left[\begin{array}{c}
u_{k} \\
z_{k+1}
\end{array}\right], \hat{y}_{k}=\left[\begin{array}{c}
y_{k} \\
z_{k}
\end{array}\right] .
$$

This closed loop dynamics ((36) and (37) with feedback (38)) are seen to be on the same form as (1) with feedback (4) and (5), hence Theorem 3.1 can also be applied for dynamic output feedback.

As before, we have to cover the different validity sets comprising the state space with ellipsoids and/or polytopes. For the augmented states, this is most easily done by not imposing restrictions on the controller state $z_{k}$, in which case the matrices defining the ellipsoids/polytopes from the static output feedback case is simply augmented with properly placed blocks of zeros.

This method prevents us from handling control input constraints as in the previous section. This can be seen from the controller parametrization; if $z_{k}$ is unbounded, then also $u_{k}$ will be unbounded (if $\hat{C}_{j} \neq 0$, that is, if the dynamic part of the controller is in effect).

Therefore, if we want to include control input constraints in the synthesis, we will need to impose bounds on the controller states, that is, we have to decide on a bounded $Z_{m}$ such that $z_{k} \in Z_{m}$, and cover $Z_{m}$ in the same manner as $X_{m}$. It is not clear to us if such a restriction in itself will introduce conservatism.

\section{A Local Solution to the Synthesis Problems}

In (Slupphaug and Foss 1999) we gave a global branch-and-bound based algorithm based on (Tuan et al. 2000) for solving the nonconvex feasibility problem associated with the controller synthesis. Herein, we present a local approach. 
The (nonconvex) feasibility problems associated with the synthesis theorems can be locally approached by using the algorithm for rank-minimization subject to LMI constraints developed in (El Ghaoui et al. 1997). To get in a position to apply that algorithm, we note that $0<P(\theta) \leqslant S^{-1} \forall \theta \in \Theta_{0}$ is equivalent to

$$
0<\hat{P}:=\left[\begin{array}{ccc}
P\left(\theta_{1}\right) & & 0 \\
& \ddots & \\
0 & & P\left(\theta_{2^{N_{0}}}\right)
\end{array}\right] \leqslant\left[\begin{array}{ccc}
S & & 0 \\
& \ddots & \\
0 & & S
\end{array}\right]=: \hat{S}^{-1},
$$

where $\theta_{1}, \ldots, \theta_{2^{N_{\varepsilon}}}$ denotes the corners (or, $\Theta_{0}$ ) of the box $\Theta$. Now, certainly $\hat{P} \leqslant \hat{S}^{-1}$, if $\hat{P}=(\eta \hat{S})^{-1}$ and $\eta>1$. Replacing the matrix inequality $\hat{P} \geqslant(\eta \hat{S})^{-1}>0$ by the LMI (Schur)

$$
\left[\begin{array}{cc}
\hat{P} & I \\
I & \eta \hat{S}
\end{array}\right] \geqslant 0
$$

where $\eta>1$ is a given constant, we may use Algorithm 1 in (El Ghaoui et al. 1997). Algorithm 1 is devised at satisfying the synthesis LMIs, while saturating the constraint (39). This gives $\hat{P}=(\eta \hat{S})^{-1}$ and hence a solution to our controller synthesis problem since $\eta>1$. In the present context where only an inequality need to be satisfied it is straightforward to find an exit condition, namely $\hat{P}_{l} \leqslant \hat{S}_{l}^{-1}$ ( $l$ denotes the iteration index). If equality was needed as in (El Ghaoui et al. 1997) it would not be as easy to find an exit condition. However, for the more structured problem in (El Ghaoui et al. 1997) this can be done (El Ghaoui et al. 1997, Theorem 2.3).

Before we present the algorithm we will show that there are LMI feasibility problems providing nontrivial necessary conditions for the nonconvex synthesis problems. Now, if there are feasible $S^{*}$ and $P^{*}$ to the synthesis LMIs with $P^{*}(\theta) \leqslant S^{*-1} \forall \theta \in \Theta$ (or, $S^{*} \leqslant P^{*}(\theta)^{-1} \forall \theta \in \Theta$ ), it can be seen that $S$ satisfying $S^{*} \leqslant S=\min _{\theta \in \Theta} P^{*}(\theta)^{-1}$ also solves the problem. Also, $\min _{\theta \in \Theta} P^{*}(\theta)^{-1} \geqslant \eta^{-1} \max _{\theta \in \Theta} P^{*}(\theta)^{-1}$ for some finite $\eta=\eta_{\max }>1$ due to compactness of $\Theta$ and continuity of $\lambda_{\min }\left(P^{*}(\cdot)^{-1}\right)$ and $\lambda_{\max }\left(P^{*}(\cdot)^{-1}\right)$ since $P^{*}(\theta)>0 \forall \theta \in \Theta$. This means that a necessary condition for feasibility is the synthesis LMIs plus the LMI equivalent $S \geqslant\left(\eta_{\max } P(\theta)\right)^{-1} \forall \theta \in \Theta$ (or $\left.\hat{P} \geqslant\left(\eta_{\max } \hat{S}\right)^{-1}\right)$. When $N_{\theta}=0$, any $\eta_{\max } \geqslant 1$ will do. When $N_{\theta} \geqslant 1$, computing a lower bound to $\min _{\theta \in \Theta} P^{*}(\theta)^{-1}$ should be possible via LMI optimization using as viable $P^{*} \mathrm{~s}$ the ones that satisfy the LMI part. These, of course, contain the $P *$ s feasible for the total problem. The same goes for an upper bound to $\max _{\theta \in \Theta} P^{*}(\theta)^{-1}$. This issue will not be considered further herein, and we assume that a sufficiently large finite $\eta_{\max }$ is found. Thus, in our context Algorithm 1 in (El Ghaoui et al. 1997) reads:

\section{Algorithm 1'}

Step 0 Let $\eta=\eta_{\max }>1$

Step 1 Find feasible $\hat{P}_{0}$ and $\hat{S}_{0}$ for the synthesis LMIs and (39). If there are none, the problem is guaranteed infeasible, exit. If $\hat{P}_{0} \leqslant \hat{S}_{0}^{-1}$, exit. Otherwise, set $l=0$.

Step 2 Solve the LMI problem $\mathscr{P}_{1}$

$$
t_{l}:=\min \operatorname{Tr}\left(\hat{P}_{l} \hat{S}+\hat{S}_{l} \hat{P}\right)
$$

subject to all the synthesis LMIs in addition to the LMI (39) to find $\hat{P}_{l+1}$ and $\hat{S}_{l+1}$.

Step 3 If $\hat{P}_{1+1} \leqslant \hat{S}_{I+1}^{-1}$, exit. Otherwise, set $l=l+1$ and go to Step 2 . 
Theorem 4.1 The sequence $\left(t_{l}\right)_{l \geqslant 0}$ is bounded below by $2 n\left(2^{N_{\theta}} / \eta\right)$ and nonincreasing. Thus, $\left(t_{l}\right)_{l \geqslant 0}$ converges to some value $t_{o p t} \geqslant 2 n\left(2^{N_{\theta}} / \eta\right)$. Equality holds if and only if $\hat{S} \hat{P}=(1 / \eta) I$.

Proof. See (El Ghaoui et al. 1997).

We have tested Algorithm 1' on examples using $P(\theta)=P_{0}$, and although we find feasible solutions to the controller design problem for a wider range of input data (e.g. for more $R_{A}>0$ ) when using the global approach mentioned above, computations show that Algorithm l' finds a feasible solution to the controller design problem for a comparable range of input data. This is in accordance with the computations reported in (El Ghaoui et al. 1997). In addition, a solution is, if one is found, typically found much faster than is the case when using the global approach. Thus, this local algorithm seems to provide a good alternative for problems with a 'high' number of states (more than, say, 3).

Recently, other approaches to the rank-minimization problem treated in (El Ghaoui et al. 1997) have appeared, see e.g. (Apkarian and Tuan 2000b), (Fares et al. 2000). These approaches will of course also be of interest when solving the synthesis problems herein. In particular the algorithms based on sequential semidefinite programming (SSDP) seem promising (Fares et al. 2000).

\section{A set of affine APD models encapsulating a family of nonlinear systems}

In this section we will provide a procedure for obtaining the uncertainty model used in the feedback synthesis. We assume that the uncertainty is due to parameter uncertainty, and that the nonlinear structure is known. The approach consists of affinely upper- and lower bounding each of the nonlinear uncertain elements of the vector functions defining the state transition map and the output map. First, we describe how to construct the uncertainty model given these bounds. Second, we describe how to find the bounds; this involves solving a set of continuous linear semiinfinite programming problems.

\subsection{Composing the APD models given affine bounding functions}

Assume that

$$
\begin{gathered}
x_{k+1} \in \mathscr{F}\left(x_{k}, u_{k}, \Psi\right) \subset \mathbb{R}^{n} \\
y_{k} \in \mathscr{C}\left(x_{k}, \Psi\right) \subset \mathbb{R}^{r},
\end{gathered}
$$

where

$$
\begin{gathered}
\mathscr{F}(x, u, \Psi):=\{\hat{x} \mid \hat{x}=f(x, u, \psi) \text { for some } \psi \in \Psi\} \\
\mathscr{C}(x, \Psi):=\{y \mid y=h(x, \psi) \text { for some } \psi \in \Psi\}
\end{gathered}
$$

and $f, h$, and $\Psi$ are assumed to be known. Also, assume that $\forall j \in I_{n}, \hat{j} \in I_{r}, i \in I_{N_{L}}$, $(x, u, \psi) \in X_{i}^{L} \times U_{m} \times \Psi$

$$
\begin{gathered}
\underline{A}_{j}^{i} x+\underline{B}_{j}^{i} u+\underline{c}_{j}^{i} \leqslant f_{j}(x, u, \psi) \leqslant \bar{A}_{j}^{i} x+\bar{B}_{j}^{i} u+\bar{c}_{j}^{i} \\
C_{j}^{i} x+\underline{d}_{j}^{i} \leqslant h_{j}(x, \psi) \leqslant \bar{C}_{j}^{i} x+\bar{d}_{j}^{i},
\end{gathered}
$$


where $A_{j}^{i}, \underline{B}_{j}^{i}, \bar{A}_{j}^{i}, \bar{B}_{j}^{i}, C_{j}^{i}$ and $\bar{C}_{j}^{i}$ are row vectors with appropriate dimensions, while the corresponding $\underline{c}_{j}^{i} \mathrm{~s}, \bar{c}_{j}^{i} \mathrm{~s}, d_{j}^{i}$ s and $\bar{d}_{j}^{i}$ s are scalars. Superscript $i$ is introduced to separate the different local model validity sets.

Thus, for all $x \in_{i}^{L}, u \in U_{m}, \psi \in \Psi$

$$
\begin{aligned}
f(x, u, \psi)= & {\left[\begin{array}{ccc}
\theta_{1}^{i} & 0 & \cdots \\
0 & \ddots & 0 \\
\vdots & 0 & \theta_{n}^{i}
\end{array}\right]\left(\left[\begin{array}{c}
\bar{A}_{1}^{i} \\
\vdots \\
\bar{A}_{n}^{i}
\end{array}\right] x+\left[\begin{array}{c}
\bar{B}_{1}^{i} \\
\vdots \\
\bar{B}_{n}^{i}
\end{array}\right] u+\left[\begin{array}{c}
\bar{c}_{1}^{i} \\
\vdots \\
\bar{c}_{n}^{i}
\end{array}\right]\right) } \\
& +\left[\begin{array}{ccc}
1-\theta_{1}^{i} & 0 & \cdots \\
0 & \ddots & 0 \\
\vdots & 0 & 1-\theta_{n}^{i}
\end{array}\right]\left(\left[\begin{array}{c}
\underline{A}_{1}^{i} \\
\vdots \\
\underline{A}_{n}^{i}
\end{array}\right] x+\left[\begin{array}{c}
\underline{B}_{1}^{i} \\
\vdots \\
\underline{B}_{n}^{i}
\end{array}\right] u+\left[\begin{array}{c}
\underline{c}_{1}^{i} \\
\vdots \\
\underline{c}_{n}^{i}
\end{array}\right]\right)
\end{aligned}
$$

and

$$
\begin{aligned}
h(x, \psi)= & {\left[\begin{array}{ccc}
\theta_{n+1}^{i} & 0 & \cdots \\
0 & \ddots & 0 \\
\vdots & 0 & \theta_{n+r}^{i}
\end{array}\right]\left(\left[\begin{array}{c}
\bar{C}_{1}^{i} \\
\vdots \\
\bar{C}_{r}^{i}
\end{array}\right] x+\left[\begin{array}{c}
\bar{d}_{1}^{i} \\
\vdots \\
\bar{d}_{r}^{i}
\end{array}\right]\right) } \\
& +\left[\begin{array}{ccc}
1-\theta_{n+1}^{i} & 0 & \cdots \\
0 & \ddots & 0 \\
\vdots & 0 & 1-\theta_{n+r}^{i}
\end{array}\right]\left(\left[\begin{array}{c}
\underline{C}_{1}^{i} \\
\vdots \\
\underline{C}_{r}^{i}
\end{array}\right] x+\left[\begin{array}{c}
\underline{d}_{1}^{i} \\
\vdots \\
\underline{d}_{r}^{i}
\end{array}\right]\right)
\end{aligned}
$$

for some $\left(\theta_{1}^{i}, \ldots, \theta_{n}^{i}, \theta_{n+1}^{i}, \ldots, \theta_{n+r}^{i}\right) \in[0,1]^{n+r}$. Collecting the terms, we can write this as

$$
\begin{gathered}
f(x, u, \psi)=\left(A_{0}^{i}+\sum_{j=1}^{n} \theta_{j}^{i} A_{j}^{i}\right) x+\left(B_{0}^{i}+\sum_{j=1}^{n} \theta_{j}^{i} B_{j}^{i}\right) u+c_{0}^{i}+\sum_{j=1}^{n} \theta_{j}^{i} c_{j}^{i} \\
h(x, \psi)=\left(C_{0}^{i}+\sum_{j=1}^{r} \theta_{j+n}^{i} C_{j}^{i}\right) x+d_{0}^{i}+\sum_{j=1}^{r} \theta_{j+n}^{i} d_{j}^{i},
\end{gathered}
$$

where

$$
A_{0}^{i}=\left[\begin{array}{c}
A_{1}^{i} \\
A_{2}^{i} \\
\vdots
\end{array}\right] \text { and } A_{j}^{i}=\left[\begin{array}{c}
0 \\
\vdots \\
0 \\
\bar{A}_{j}^{i}-\underline{A}_{j}^{i} \\
0 \\
\vdots \\
0
\end{array}\right], j \in I_{n}
$$

and similar for the $B_{j}^{i} \mathrm{~s}, c_{j}^{i} \mathrm{~s}, C_{j}^{i} \mathrm{~s}$, and $d_{j}^{i} \mathrm{~s}, j \in\{0,1, \ldots, n\}, \hat{j} \in\{0,1, \ldots, r\}$.

Now, it follows that

$$
\begin{aligned}
\mathscr{F}(x, u, \Psi) & \subset \bigcup_{i \in \mathcal{G}_{(}(x)} \mathscr{M}_{i}(x, u, \Theta), \forall x \in X_{m}, u \in U_{m} \\
\mathscr{C}(x, \Psi) & \subset \bigcup_{i \in \mathscr{S}^{(}(x)} \mathscr{H}_{i}(x, \Theta), \forall x \in X_{m},
\end{aligned}
$$


where the $\mathscr{M}_{i} \mathrm{~s}\left(\mathscr{H}_{i} \mathrm{~s}\right)$, see (2) and (3), are defined by the above given $A_{j}^{i} \mathrm{~s}, B_{j}^{i} \mathrm{~s}$, and $c_{j}^{i} \mathrm{~s}$ $\left(C_{j}^{i} \mathrm{~s}\right.$ and $\left.d_{j}^{i} \mathrm{~s}\right)$, and $\Theta=[0,1]^{n+r}$. This means that the uncertain system associated with $\mathscr{F}$ is contained in the uncertain system associated with the $\mathscr{M}_{i}$ s, and likewise for the uncertain output map. Thus, if we can stabilize some point $x_{s}$ of the latter uncertain system using feedback from the output, we will at the same time stabilize it for $\mathscr{F}$ (cf. Section 3).

Next, we describe one way to find these affine bounding functions.

\subsection{Finding the upper and lower bounds}

We will now show that finding the $N_{L}(n+r)$ upper bounding and $N_{L}(n+r)$ lower bounding scalar affine functions can be formulated as $2 N_{L}(n+r)$ continuous linear semi-infinite programs (Goberna and López 1998). These are proposed solved by a series of ordinary (finite) linear programs (LPs). We only show the upper bounding for the elements of $f$, as the other bounding problems are essentially equal.

The affine upper bounding function for state equation $j$ in local model validity set $i$ should approximate as closely as possible the nonlinear uncertain function from above. Let $\forall j \in\{1, \ldots, n\}$

$$
g_{j}(x, u):=\max _{\psi \in \Psi} f_{j}(x, u, \psi) \forall(x, u) \in X_{i}^{L} \times U_{m},
$$

then this can be transformed into the following linear semi-infinite optimization problem

$$
V^{*}:=\min _{p:=\left(\bar{A}_{j}^{\bar{B}}, \bar{B}_{j}^{i}, \bar{c}_{j}^{\prime}\right)} L(p):=\int_{\boldsymbol{X}_{i}^{L} \times U_{m}} \ldots \int_{A_{j}^{i}} x+\bar{B}_{j}^{i} u+\bar{c}_{j}^{i}-g_{j}(x, u) d x d u
$$

subject to $\forall z \in Z:=X_{i}^{L} \times U_{m}$

$$
h(p ; z):=\bar{A}_{j}^{i} x+\bar{B}_{j}^{i} u+\bar{c}_{j}^{i}-g_{j}(x, u) \geqslant 0,
$$

where $z=(x, u)$. Note that, assuming the multiple integral in (40) exists, the cost function $L$ is indeed linear in the unknowns $p$. However, we have an uncountable number of linear constraints parameterized in $z$.

By using a well known fact from calculus we can conservatively transform this uncountable number of linear constraints into a finite number of linear constraints. From calculus we know that if $\tilde{h} \in C^{2}$ where $\hat{h}: Z \rightarrow \mathbb{R}$ and $Z$ convex, then for all $w, z \in Z$

$$
\tilde{h}(w)=\tilde{h}(z)+\nabla \tilde{h}(z)^{T}(w-z)+\frac{1}{2}(w-z)^{T} \nabla^{2} \tilde{h}(z+t(w-z))(w-z)
$$

for some $t \in(0,1)$. Meaning that for all $z, w \in Z$

$$
\left|\tilde{h}(z)+\nabla \tilde{h}(z)^{T}(w-z)-\tilde{h}(w)\right| \leqslant \frac{n+m+1}{2} \gamma\|w-z\|_{\infty}^{2}
$$

if $\left\|\nabla^{2} \tilde{h}(z)\right\|_{i \infty} \leqslant \gamma$ for all $z \in Z$. This follows from the Hölder inequality, that $\|z\|_{2} \leqslant \sqrt{n+m+1}\|z\|_{\infty}$, and that $\|G\|_{i \infty}:=\max _{x \neq 0}\left(\|G x\|_{\infty} /\|x\|_{\infty}\right)$ for any matrix $G$.

Now, define a finite set $\mathscr{G}(\varepsilon)$ (associated with $Z$ ) denoted an $\varepsilon$-grid that satisfies $\forall z \in Z \exists\left\{w_{1}, \ldots, w_{n+m+1}\right\} \subset \mathscr{G}(\varepsilon)$ such that $z \in C o\left\{w_{1}, \ldots, w_{n+m+1}\right\}$ and $\left\|w_{i}-w_{j}\right\| \leqslant$ $\varepsilon \forall i, j \in I_{n+m+1}$. In the following we will for simplicity assume that $Z$ is such that 
$\varepsilon$-grids exist for it satisfying $\mathscr{G}(\varepsilon) \subset Z$ for all $\varepsilon>0$. Note that e.g. a standard $\varepsilon$-grid on a box satisfies this when the border (or, box surface) is gridded as well.

Lemma 5.1 Assume $g_{j} \in C^{2}, Z$ convex, and $\left\|\nabla^{2} g_{j}(z)\right\|_{i \infty} \leqslant \gamma$ for all $z \in Z$. Then it holds that, if for some $p \in \mathbb{R}^{n+m+1}$

$$
h(p ; z) \geqslant \frac{n+m+1}{2} \gamma \varepsilon^{2} \forall z \in \mathscr{G}(\varepsilon)
$$

then

$$
h(p ; z) \geqslant 0 \forall z \in Z \text {. }
$$

Proof. Take an arbitrary $z \in Z$. By the definition of an $\varepsilon$-grid, $z \in \operatorname{Co}\left\{w_{1}, \ldots, w_{n+m+1}\right\}$ for some $n+m+1$-tuple $\left\{w_{1}, \ldots, w_{n+m+1}\right\}$ in $\mathscr{G}(\varepsilon)$ satisfying $\left\|w_{i}-w_{j}\right\|_{\infty} \leqslant \varepsilon \forall i$, $j \in I_{n+m+1}$. Now, from (41) we get

$$
\left|g_{j}(z)+\nabla g_{j}(z)^{T}(w-z)-g_{j}(w)\right| \leqslant \frac{n+m+1}{2} \gamma \varepsilon^{2} \forall w \in C o\left\{w_{1}, \ldots, w_{n+m+1}\right\} .
$$

Note that in particular this holds for $w \in\left\{w_{1}, \ldots, w_{n+m+1}\right\}$. By assumption

$$
h(p ; w) \geqslant \frac{n+m+1}{2} \gamma \varepsilon^{2} \forall w \in\left\{w_{1}, \ldots, w_{n+m+1}\right\} .
$$

Thus,

$$
h(p ; z) \geqslant g_{j}(z)+\nabla g_{j}(z)^{T}(w-z)-g_{j}(w) \forall w \in\left\{w_{1}, \ldots, w_{n+m+1}\right\},
$$

or

$$
\left[\begin{array}{ll}
\bar{A}_{j}^{i} & \bar{B}_{j}^{i}
\end{array}\right] w+\bar{c}_{j}^{i} \geqslant g_{j}(z)+\nabla g_{j}(z)^{T}(w-z) \forall w \in\left\{w_{1}, \ldots, w_{n+m+1}\right\} .
$$

By affinity (42) holds for any $w \in C o\left\{w_{1}, \ldots, w_{n+m+1}\right\}$, and in particular for $w=z$ giving

$$
h(p ; z)=\left[\begin{array}{ll}
\bar{A}_{j}^{i} & \bar{B}_{j}^{i}
\end{array}\right] z+\bar{c}_{j}^{i}+g_{j}(z) \geqslant 0
$$

and the result follows from the arbitrariness of $z$.

Based on this we propose the following algorithm for solving the linear semiinfinite optimization problem (40).

\section{Algorithm 5.1 (Affine upper approximation)}

Step 1 If $\gamma=0, g_{j}$ is affine-stop. Pick any $\varepsilon_{0}, \beta>0$ and $0<\kappa_{1}<1$. Let $l=0$.

Step 2 Let $\xi_{l}=((n+m+1) / 2) \gamma \varepsilon_{l}^{2}$. Solve the associated LP defined by $L(p)$, $h(p ; w) \geqslant \xi_{l}, \forall w \in \mathscr{G}\left(\varepsilon_{l}\right)$. Let $V_{L P}$ denote the optimum value and $p^{*}$ the corresponding optimum point. Check so-called $\beta$-optimality by

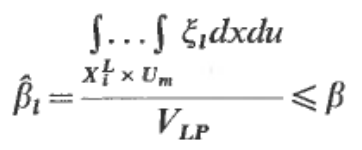

if so, stop. If not, go to Step 3.

Step 3 Let $l=l+1$, and let $0<\varepsilon_{l}<\kappa_{1} \varepsilon_{l-1}$, return to Step 2 . 
If Algorithm 5.1 halts we have a $\beta$-optimal solution. This means that the optimal value $V^{*}$ is greater than or equal to $V_{L P}$ minus $\beta V_{L P}$, i.e. $V^{*} \geqslant V_{L P}(1-\beta)$ upon exit.

Algorithm 5.1 terminates in finite time as proved next.

Theorem 5.1 Assume $g_{j} \in C^{2}, Z$ convex, and $\left\|\nabla^{2} g_{j}(z)\right\|_{i \infty} \leqslant \gamma$ for all $z \in Z$. Then, the above algorithm terminates in finite time with a $\beta$-optimal solution.

Proof. It follows from the assumptions, Lemma 5.1, and the definition of $\mathscr{G}$ that the associated LPs indeed have a finite number of inequalities and that they provide upper bounds $\bar{V}^{*}=V_{L P}$ to $V^{*}$. A lower bound $\underline{V}^{*}$ at iteration $l$ on $V^{*}$ can be computed by

$$
\underline{V}^{*}=V_{L P}-\int_{X_{i}^{L} \times U_{m}} \ldots \int_{l} \xi_{l} d x d u .
$$

$V^{*}$ is the optimum that would be obtained if the right-hand side of the constraints in the LP were lowered by $\xi_{l}$ giving 0 , this can be seen from the Karush-Kuhn-Tucker conditions for this specially structured LP problem (the associated optimum point would be the same as $p^{*}$ apart from the last element $\bar{c}_{j}^{i *}$ that would be lowered by $\xi_{l}$ ). Since only a finite number of constraints are considered, it provides a lower bound on $V^{*}$. Now, $\beta$-optimality would follow if

$$
\frac{\bar{V}^{*}-\underline{\underline{V}}^{*}}{\bar{V}^{*}} \leqslant \beta .
$$

This is exactly the $\beta$-optimality check given in the algorithm, and hence a $\beta$-optimal solution results if it halts. When it comes to proving that the algorithm indeed will terminate in finite time, we note that $\lim _{l \rightarrow \infty} \xi_{l}=0$. Also $V_{L P}>\kappa_{2}>0$ since $g_{j}$ is not affine due to that step 2 is reached, thus the $\beta$-optimality check will hold true at some finite iteration.

From a practical point of view, the size of the involved LPs is of importance, and fairly large scale problems do indeed result as is shown in the example below. We have not looked closely into how to alleviate these problems, but gridding with different density, according to the Hessian information, in different parts of the local model validity set is one obvious possibility.

It must also be mentioned, that the problem of finding $g_{j}$ may be hard. In some simple real world related examples, however, it has been straightforward since the maximizing $\psi$ has been constant over quadrants.

Finally we remark that utilizing a bound on the gradient instead of a bound on the Hessian results in typically quadratically more grid points. In addition the associated algorithm will be more complex since the gradient of $h$ depends on the optimization variable $p$.

We conclude this section with an application of the proposed algorithm.

\section{Example 1 (Upper approximation)}

Consider the affine upper approximation of

$$
g\left(x_{1}, x_{2}\right)=x_{1}^{3} \exp \left(-x_{2}\right) \sin \left(4 \pi x_{2}\right)
$$




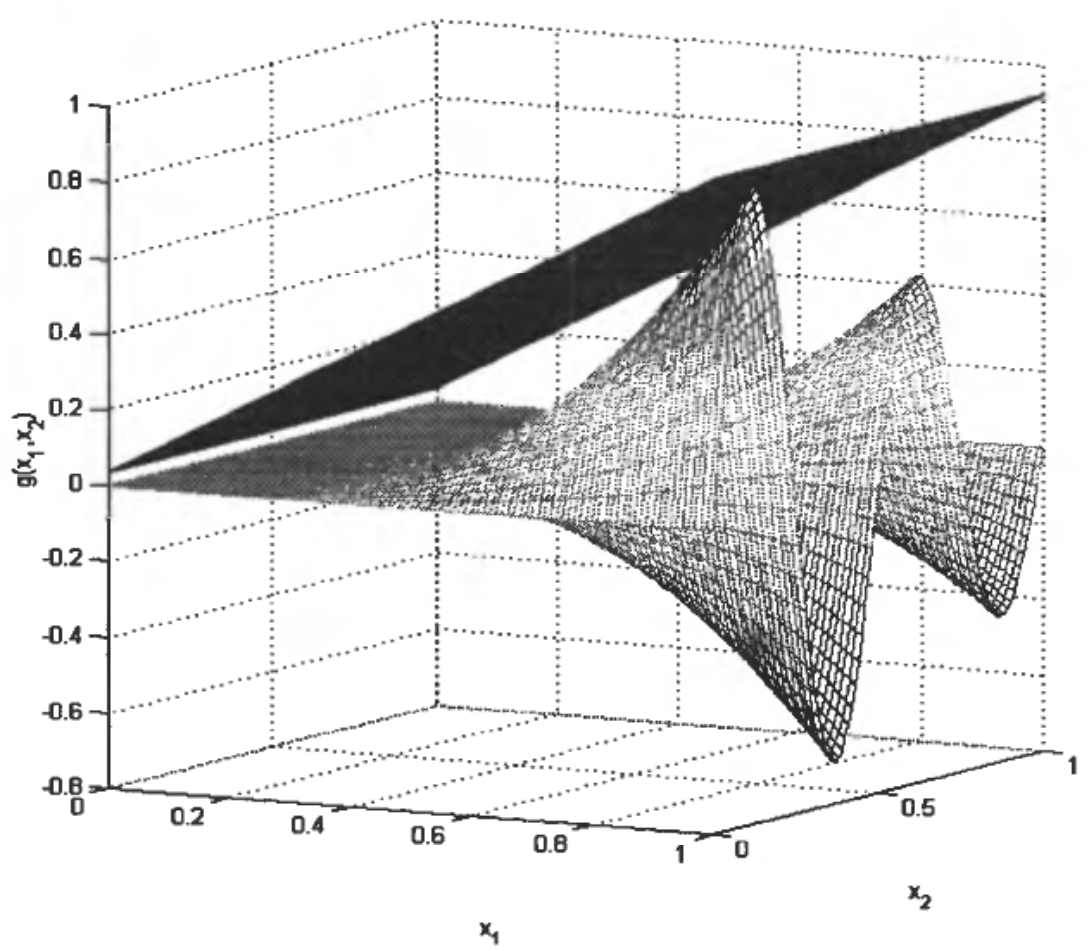

Figure 1. $g\left(x_{1}, x_{2}\right)=x_{1}^{3} \exp \left(-x_{2}\right) \sin \left(4 \pi x_{2}\right)$ and a 0.1-optimal affine upper bounding function found using Algorithm 5.1.

over [0 1] $0\left[\begin{array}{ll}0 & 1\end{array}\right]$. It can be shown that for all $\left(x_{1}, x_{2}\right) \in\left[\begin{array}{ll}0 & 1\end{array}\right] \times\left[\begin{array}{ll}0 & 1\end{array}\right]$

$$
\left\|\nabla^{2} g_{j}\left(x_{1}, x_{2}\right)\right\|_{i \infty \infty} \leqslant 16 \pi^{2}-1+8 \pi+3(1+4 \pi) \approx 223 .
$$

We initialized with $\varepsilon_{0}=1, \beta=0.05$ and $\kappa_{1}=0.9$, and used a standard $\varepsilon$-grid technique to generate $\mathscr{G}(\varepsilon)$. We let $\varepsilon_{l}=\kappa_{1} \varepsilon_{l-1}$. Each LP was initialized with the solution at the previous iteration. This resulted in the 0.1-optimal upper approximation shown in Figure 1. We used MATLAB and the LP solver e04mbf.m from NAG for implementation of the algorithm. On a $450 \mathrm{MHz} P C$ with $256 \mathrm{MB} R A M$ it took about 2 seconds to find a 0.1 -optimal solution. The returned solution was $p^{*}=(0.88530 .00000 .0388)$, and the last used $\varepsilon$ was $\varepsilon_{44}=0.0108$ which results in about 8600 inequalities in the associated (last and largest) $L P$.

\section{Robust Nonlinear Model Predictive Control}

We will in this section show how we can use the precomputed matrix function $P(\theta)=P_{0}+\Sigma_{j}^{N_{\underline{\theta}}} \theta_{j} P_{j}$, the matrix $M$, and the feedback matrices $\left\{K_{j}\right\}_{j=1}^{M_{L}}$, $\left\{k_{j}\right\}_{j}^{M_{L} M_{M_{L}+1}^{o}}$ from the state-feedback version of Theorem 3.2 in an MPC scheme to guarantee robust stability. This is done by adding the hard constraint to the MPC optimization problem that the Lyapunov function $V\left(x_{k}, k\right)=x_{k}^{T} P\left(\theta_{k}\right) x_{k}$ should decrease at each time step. This MPC problem is feasible at each time-step if $x_{k}$ is in the region of attraction associated with Theorem 3.2, since an input trajectory based on the feedback matrices $\left\{K_{j}\right\}_{j=1}^{M_{L}},\left\{k_{j}\right\}_{j=M_{M_{L}+1} M^{o}}$ then provides a feasible initial point in the optimization. Consult (Slupphaug and Foss 1999) for the same approach using a parameter independent Lyapunov function, and for more extensive comments. 
Define an MPC optimality criterion on the prediction horizon $N$ :

$$
\begin{gathered}
\phi\left(\pi_{k}, \chi_{k} ; k, x_{k}, u_{k-1}\right) \\
\pi_{k}=\left\{u_{k}, \ldots, u_{k+N-1}\right\} ; \chi_{k}=\left\{x_{k+1}, \ldots, x_{k+N}\right\}, \\
\phi: \mathbb{R}^{m} \times \ldots \times \mathbb{R}^{m} \times \mathbb{R}^{n} \times \ldots \times \mathbb{R}^{n} \times \mathbb{N} \times \mathbb{R}^{n} \times \mathbb{R}^{m} \rightarrow \mathbb{R}_{+}
\end{gathered}
$$

Based on this, we can specify the following optimization problem, denoted $\mathscr{P}_{M P C}$, to be found feasible or solved at each time step $k$ :

$$
\min _{\pi_{k} \in \Pi} \phi\left(\pi_{k}, \chi_{k}^{n o m} ; k, x_{k}, u_{k-1}\right)
$$

subject to the hard constraints

$$
\left\|A^{i}\left(\theta_{k}\right) x_{k}+B^{i}\left(\theta_{k}\right) u_{k}+c^{i}\left(\theta_{k}\right)\right\|_{P\left(\theta_{k+1}\right)}^{2}-\left\|x_{k}\right\|_{P\left(\theta_{k}\right)}^{2} \leqslant-\left\|x_{k}\right\|_{M}^{2}, \forall \theta_{k}, \theta_{k+1} \in \Theta_{0}, i \in \mathscr{I}\left(x_{k}\right)
$$

and the soft constraints

$$
\chi_{k}^{\text {nom }} \subset X \times \ldots \times X \text {. }
$$

$P(\theta)$ and $M$ are given from Theorem 3.2, and $\Pi=U_{m} \times \ldots \times U_{m} \cdot \chi_{k}^{n o m}$ denotes the prediction on the $N$ step ahead horizon, using some (possibly nonlinear) nominal model which typically, when restricted to $X_{m} \times U_{m} \times \Psi$, is within the model uncertainty class. The so-called soft constraints are defined by $X$. These should be chosen such that $X \subset X_{m}$, since this will 'softly' force the state to be within the state constraints $X_{m}$. If the nominal model is within the assumed model uncertainty class, and the region of attraction $\left\{x \mid \exists \theta, x^{T} P(\theta) x \leqslant \alpha(\theta)\right\}$ is contained in $X$, then the soft constraints can be satisfied for every $k \geqslant 0$ if the initial point $x_{0}$ is within the region of attraction. If the choice of $X$ or the choice of nominal model makes some of the soft constraints infeasible at some $k$, they can be dropped (thereby the name soft constraint) while retaining the closed-loop plant states within $X_{m}$ (in fact, within $\left\{x \mid \exists \theta, x^{T} P(\theta) x \leqslant\right.$ $\alpha(\theta)\})$. This follows from the hard constraints.

The solution procedure for the MPC is defined as follows:

Step 1 At time step $k$, the initial choice for $\pi_{k}$ in the assumed feasible iterative optimization algorithm is computed by the precomputed state-feedback (4), (5), $\left\{K_{j}\right\}_{j=1}^{M_{L}},\left\{k_{j}\right\}_{j=M_{L+1} M_{L}}$ derived from Theorem 3.2, using the nominal model for prediction.

Step 2 The iterative optimization algorithm for solving $\mathscr{P}_{M P C}$ is run until convergence or, alternatively, terminated earlier.

We are now ready to state the following:

Theorem 6.1 (Nonlinear Robust MPC) Assume that the system to be controlled is contained in the assumed model uncertainty class, that is, given a state $x_{k} \in X_{m}$ and an input $u_{k} \in U_{m}$ the next state $x_{k+1} \in U_{i \in \mathscr{G}\left(x_{k}\right)} \mathscr{H}_{i}\left(x_{k}, u_{k}, \Theta\right)$. Then an MPC based on the solution procedure above renders the origin of the closed loop system affinely quadratically stable (cf. definition 3.1) with a region of attraction associated with $\left\{x \mid x^{T} R_{A} x \leqslant 1\right\}$ of at least $\left\{x \mid \exists \theta \in \Theta, x^{T} P(\theta) x \leqslant \alpha(\theta)\right\} \subset X_{m}$, and the control input constraints, $U_{m}$, are satisfied on all closed loop trajectories starting within $\left\{x \mid \exists \theta \in \Theta, x^{T} P(\theta) x \leqslant \alpha(\theta)\right\}$. 
Proof. First, note that all $u_{k}$ computed from the feasible (see below) MPC can be written as $u_{k}=\tilde{K}_{k} x_{k}+\tilde{k}_{k}$, for some time-varying $\tilde{K}_{k}$ and $\tilde{k}_{k}$. The reason that we emphasize this obvious relation, is to make the MPC closed-loop fit into the stability framework of Section 3. Also, we need the additional observation that we could have assumed the feedback matrices to be time-varying in all transitions of the proof of Theorem 3.1. To see that the hard constraints (43), which are guaranteed to be satisfied upon exit from Step 2, implies the condition in definition 3.1, and thereby affine quadratic stability, we have to replace $\Theta_{0}$ with $\Theta$ in (43). This can be done by using the same steps as in the proof of Theorem 3.1. Equation (43) can essentially be written on the same form as (22)/(23). By going from these equations to the end of the theorem, and back to the outset, realizing that all the needed transitions are equivalences (that is, we do not need the transitions based on the S-procedure, and because of the state feedback, after Schur complements all equations are affine in the parameters and hence we can replace $\Theta_{0}$ with $\Theta$ without conservatism), we arrive at the definition of affine quadratic stability.

Finally, Step 1 of the solution procedure will always provide a feasible $\pi_{k} \in \Pi$ satisfying the hard constraints when $x_{k} \in\left\{x \mid \exists \theta, x^{T} P(\theta) x \leqslant \alpha(\theta)\right\}$, this follows directly from Theorem 3.2.

The solution procedure utilizes an iterative optimization algorithm. We have assumed that this is a so-called feasible algorithm meaning that if the initial choice satisfies the hard constraints, the consecutive iterations will also satisfy these constraints. Thus, the iterative search improves the nominal performance within the frame of guaranteed robust stability. As a consequence, the iterative algorithm may be terminated prior to convergence (for example because of limited computation time) without affecting the stability. Further, possible non-convexity of the optimization problem (which e.g. could be caused by a non-linear nominal prediction model) does not affect the stability result either. Also, any prediction horizon $N$ can be used without affecting stability. Finally, it is important to notice that the minimum required on-line computational load is very low. An example of application of this kind of nonlinear constrained robust MPC can be found in (Slupphaug and Foss 1998).

\section{Concluding remarks}

We have developed a rigorous method for computing a robustly stabilizing piecewise affine output feedback controller for constrained nonlinear discrete-time systems with uncertain, possibly time-varying, parameters. Moreover, the method supports control input and state constraints. The method, which depends on obtaining a feasible solution to a set of LMIs subject to one spectral radius constraint on the product of two positive definite matrices, extends earlier results since the Lyapunov matrix is parameter dependent. By this it is possible to exploit implicitly given (via the sampling rate and the parameters' bounds) information on the bounds of the parameters' rate-of-variation. Further, output feedback, as opposed to earlier results treating the state-feedback case, is considered.

The design method will in its most general form only be solvable for very lowdimensional systems due to that it results in possibly very large scale optimization problems. However, if parts of the uncertain system is linear and known, this can be exploited and may significantly reduce the size of the associated optimization problems. The system may for example contain pure integrators, or the output may simply be a subset of the states. 
A local solution algorithm for solving the synthesis problem is proposed. Also, nontrivial necessary conditions in the form of LMIs are described for the synthesis problems.

The uncertainty model that is considered is a piecewise affine parameter dependent discrete-time state-space model. It is shown how the model class can be constructed from a nonlinear partially unknown state-space system. The construction method is based on the solution of a set of continuous linear semi-infinite programs using a grid discretization approach.

Finally, an on-line computationally feasible nonlinear MPC algorithm with robust stability properties is derived by using the result in Theorem 3.2. This result is limited to the state-feedback case.

Important future issues related to this work are improved methods for developing the uncertainty models, developing the design method to further reduce complexity, and applying the methodology to examples of different complexity. Exploiting explicitly given bounds on the parameters' rate-of-variation as well as improving the local solution strategy are also natural topics to investigate further. Finally, extending the MPC result to output-feedback is also a very challenging problem.

\section{Notes}

1. Note that affine parameter dependent mean that the model matrices are affine in the parameter vector $\theta$. Since the models are also affine, we get affine parameter dependent models. We will concatenate this to affine APD models.

2. If tighter approximations are needed, one can use unions of polytopes or ellipsoids. This extension is trivial (Slupphaug 1998).

3. $\|x\|_{H}:=\sqrt{x^{T} H x}, H>0$.

4. $N(\varepsilon):=\{x \mid\|x\| \leqslant \varepsilon\}$.

5. The condition that $W^{\prime}(\theta)$ must have non-negative elements, i.e. be an element of $\mathbb{R}^{n_{f_{1}} \times n_{E_{i}}}$, can be expressed as an LMI condition on each of the elements of $W^{l}(\theta)$.

6. Since the equations containing both $\theta_{k}$ and $\theta_{k+1}$ now become decoupled, we replace $\theta_{k} \in \Theta$ and $\theta_{k+1} \in \Theta$ with $\theta \in \Theta$. It is also noteworthy that this is the step where one looses the possibility to exploit explicitly given bounds on the parameters' rate-of-variation.

7. Here, we need that the matrix inequalities are multi-concave. The conditions for this are obtained by reversing the signs in the conditions for multi-convexity.

8. As noted before, we could have used unions of ellipsoids.

\section{REFERENCES}

ACKermann, J. (1993). Robust Control: Systems with Uncertain Parameters. Springer.

Allgöwer, F., Badgwell, T. A., QIN, J. S., Rawlings, J. B. and Wright, S. J. (1999). Nonlinear Predictive Control and Moving Horizon Estimation-An Introductory Overview. In: Advances in Control: Highlights of ECC'99 (Paul M. Frank, Ed.). Springer.

APKARIAN, P. and TuAN, H. D. (2000a). Parameterized LMIs in Control Theory. SIAM J. on Control and Optimization 38(7), pp. 1271-1264.

ApKarian, P., Becker, G., Gahinet, P. and Kajiwara, H. (1996). LMI Techniques in Control Engineering from Theory to Practice. Workshop Notes CDC 1996, Kobe, Japan. Copies can be obtained on request to the authors of the present paper.

Apkarian, P. and Hoang Duong Tunn (2000b). Robust Control via Concave MinimizationLocal and Global Algorithms. IEEE Transactions on Automatic Control 45(2), pp. 299305.

Bemporad, A. and Morari, M. (1999). Robust Model Predictive Control: A Survey. In: Robustness in Identification and Control (A. Garului, A. Tesi and A. Vicino, Eds). Vol. 245 of Lecture Notes in Control and Information Sciences. Springer-Verlag.

Boyd, S., El Ghaoui, L., Feron, E. and Balakrishnan, V. (1994). Linear Matrix Inequalities 
in System and Control Theory, Number 15. In: SIAM Studies in Applied Mathematics. SIAM.

El Ghaoui, L., Oustry, F. and AitRami, M. (1997). A Cone Complementary Linearization Algorithm for Static Output-Feedback and Related Problems. IEEE Transactions on Automatic Control 42(8), pp. 1171-1176.

Fares, B., APKARIAN, P. and Noll, D. (2000). An Augmented Lagrangian Method for a class of LMI-Constrained Problems in Robust Control Theory. Submitted to Int. Journal of Control.

Gahinet, P., Apkarian, P. and Chilali, M. (1996). Affine Parameter-Dependent Lyapunov Functions and Real Parametric Uncertainty. IEEE Transactions on Automatic Control 41(3), pp. 436-442.

Goberna, M. A. and López, M. A. (1998). Linear Semi-Infinite Optimization, Number 2. In: Mathematical Methods in Practice. Wiley.

Goh, K. C., Safonov, M. G. and Papavassilopoulos, G. P. (1994). A Global Optimization Approach for the BMI Problem. In: Proceedings of the 33rd CDC, Lake Buena Vista, FL, USA. pp. 2009-2014.

GoH, K. C., SAFOnOv, M. G. and Ly, J. H. (1996). Robust Synthesis via Bilinear Matrix Inequalities. International Journal of Robust and Nonlinear Control 6(9-10), pp. 10791095.

Hassibi, A. and Boyd, S. (1998). Quadratic Stabilization and Control of Piecewise-Linear Systems. In: Proceedings American Control Conference, Philadelphia, PA, USA.

HoRn, R. A. and Johnson, C. R. (1992). Matrix Analysis. Cambridge University Press. ISBN 0-521-38632-2.

Johansson, M. (1999). Piecewise Linear Control Systems. PhD thesis. Lund Institute of Technology.

Morari, M. and Lee, J. H. (1997). Model Predictive Control: Past, Present and Future. In: Puhlished at the Joint 6th Intern. Symp. on Process Systems Engineering and 30th European Symp. on Computer Aided Process Engineering (PSE'97-ESCAPE-7), Vol. 21. Pergamon.

Nesterov, Y. and NemirovskiI, A (1994). Interior-point Polynomial Algorithms in Convex Programming. Vol. 13 of Studies in Applied Mathematics. SIAM. Philadelphia, PA.

QIN, S. J. and BADGWELL, T. A. (1997). An Overview of Industrial Model Predictive Control Technology. In: Fifth International Conference on Chemical Process Control (J. C. KanToR, C. E. Garcia and B. Carnahan, Eds). AIChE Symposium Series 316. pp. 232-256.

QIN, S. J. and BADGWELL, T. A. (1999). An overview of nonlinear model predictive control applications. In: Nonlinear Predictive Control (F. Allgöwer and A. ZHENG, Eds). Birkhäuser, Basel. In press.

SCHERER, C. and WeIland, S. (1999). Lecture Notes DISC Course on Linear Matrix Inequalities in Control. *http://www.er.ele.tue.nl/sweiland/lmi99.htm.

Skogestad, S. and Postlethwarte, I. (1996). Multivariate Feedback Control. John Wiley \& Sons, Chichester, England.

Slupphaug, O. and Foss, B. A. (1998). Bilinear Matrix Inequalities and Robust Stability of Nonlinear Multi-Model MPC. In: Proceedings of the American Control Conference. Philadelphia, PA, USA.

Slupphaug, O. and Foss, B. A. (1999). Constrained Quadratic Stabilization of Discrete-Time Uncertain Nonlinear Multi-Model Systems using Piecewise Affine State-Feedback. Int. J. of Control 72(7/8), pp. 686-701.

SluppHaUG, O. (1998). On Robust Constrained Nonlinear Control and Hybrid Control: BMIand MPC-based State-Feedback Schemes. PhD thesis. Norwegian University of Science and Technology, Department of Engineering Cybernetics. *http://www.itk. ntnu.no/dr.avhandlinger/1998/.

Tuan, H. D., Apkarian, P., Hosoe, S. and Tuy, H. (2000). d.c. optimization approach to robust control: feasibility problems. International Journal of Control 73(2), pp. 89-104.

Wolkowicz, H., Saigal, R. and Vandenberghe, L. (Eds) (2000). Handbook of Semidefinite Programming. Vol. 27 of International Series in Operations Research and Management Science. Kluwer, Boston, MA. 